\title{
Robust Multidimensional Poverty Comparisons
}

\author{
by \\ Jean-Yves Duclos \\ Department of Economics and CRÉFA, Université Laval, Canada, \\ and Institut d'Anàlisi Econòmica (CSIC), UAB, Barcelona, Spain \\ David Sahn \\ Food and Nutrition Policy Program, Cornell University \\ and \\ Stephen D. Younger \\ Food and Nutrition Policy Program, Cornell University
}

\begin{abstract}
We investigate how to make poverty comparisons using multidimensional indicators of well-being, showing in particular how to check whether the comparisons are robust to the choice of poverty indices and poverty lines. Our methodology applies equally well to either of what can be defined as "union" and "intersection" approaches to dealing with multidimensional indicators of well-being. When one of two variables is discrete, our methods specialize to those that Atkinson (1991), Jenkins and Lambert (1993) and others have developed to deal with household composition heterogeneity. The results also extend the statistical results recently derived in Davidson and Duclos (2000) to cases where well-being is measured in two or more dimensions. We thus derive the sampling distribution of various multidimensional poverty estimators, including estimators of the "critical" frontiers of poverty lines above which multidimensional poverty comparisons are no longer ethically robust.
\end{abstract}

Keywords Multidimensional Poverty, Stochastic Dominance. JEL \# D31,D63,I31,I32.

Duclos' research was supported by grants from SSHRC and the MIMAP programme of the Canadian IDRC. Sahn's and Younger's participation was supported by the US Agency for International Development and the African Economic Research Consortium. An early version of this work was written as a supporting paper for the World Development Report (2000), regarding which we are grateful to Christian Grootaert for his comments and support. We also thank Rob Strawderman and Steve Westin for technical advice, and Nicolas Beaulieu and Wilson Perez for their excellent research assistance.

Corresponding addresses:

Jean-Yves Duclos: Département d'économique, Pavillon de Sève, Université Laval, Québec, Canada, G1K 7P4; Tel.: (418)656-7096; Email: jduc@ecn.ulaval.ca.

David Sahn and Stephen Younger: Room 3M12 MVR Hall, Cornell University, Ithaca, NY15843. E-mail: des16@cornell.edu or sdy1@cornell.edu.

August, 2001 


\section{Introduction}

It is common to assert that poverty is a multi-dimensional phenomenon, yet most empirical work on poverty uses a one-dimensional yardstick to judge a person's well-being, usually expenditures or income per capita or per adult equivalent. When more than one indicator of well-being is used, poverty comparisons are either made for each indicator independently of the others, or are performed using an arbitrarily-defined aggregation of the multiple indicators into a single index. In either case, aggregation across individuals of individual poverty statuses requires a poverty index, and no single such index has been devised that has received unanimous approval. Multidimensional poverty comparisons also require estimation of multidimensional poverty lines, a procedure which is ethically and empirically problematic even in a unidimensional setting.

Taking as a starting point our belief that multidimensional poverty comparisons are theoretically attractive, our purpose in this paper is to address some of their methodological, empirical, and statistical difficulties. In particular, we show how to determine whether truly multidimensional poverty comparisons are robust to the aggregation of multiple indicators, robust to the selection of multidimensional poverty lines and of multidimensional poverty indices, and robust to the presence of sampling variability in the estimators used.

We start in section 2 by considering poverty comparisons that involve two or more measures of well-being, and by asking whether poverty is lower in population A than in population B. One can think of poverty being defined on many dimensions, along the lines of Sen's "capabilities" approach to poverty (see for instance Sen (1985)), and we test jointly along all of them. Here, we make an important distinction between intersection and union definitions of poverty. In one of the few papers on multidimensional poverty measurement, Bourguignon and Chakravarty (1998) argue that, if we measure well-being in the dimensions of income and height, say, then a person should be considered poor if her income falls below the income poverty line or if her height falls below the height poverty line. We may define this as a union definition of multidimensional poverty. An intersection definition, however, would consider a person to be poor only if she falls below both poverty lines. The tests that we develop are applicable to both definitions.

Section 3 presents a different approach to multidimensional poverty comparisons. Rather than asking "Is poverty lower for A than B over all reasonable poverty lines in all dimensions?" we ask, "What is the range of poverty lines in all dimensions over which we can be sure that poverty is lower for A than for B?" This approach eliminates the need to make an arbitrary choice of "reasonable" limits for the range of poverty lines.

In section 4, we show how our methodology specializes to cases in which one of the measures of well-being is discrete. For example, we might consider literacy as one indicator of well-being, and believe for instance that literate people are better off than illiterate people of the same income level. In such cases, we can split the total population into sub-groups using the value of the discrete variable of well-being, and compare the levels of a continuous variable of well-being such as income within and across subgroups. This turns out to be just a special case of the methods derived in section 2. Exactly 
the same method applies to poverty comparisons in which a dimension (e.g., incomes) contributes to well-being, but does so differently for those belonging to different subgroups of the population. Group membership defines the other dimension of well-being. Again, the rationale for this is that each sub-group can reasonably be expected to have a different level of total well-being for the same amount of income. Atkinson (1991) and Jenkins and Lambert (1993) (and more recently in Chambaz and Maurin (1998) and Duclos and Makdissi (2000) for instance) developed this type of analysis for poverty comparisons in the presence of household heterogeneity in order to avoid relying on the choice of one particular set of equivalence scales when making poverty comparisons.

Throughout, we follow the dominance approach to poverty comparisons, as initially developed in Atkinson (1987) and Foster and Shorrocks (1988a,b,c) ${ }^{1}$. It is well-known that one important advantage of this approach is that it is capable of generating poverty orderings that are robust to the specification of poverty lines over broad ranges. Given the sensitivity of most poverty measures to the poverty line, this is an important consideration. As we show, this approach can also ensure robustness to the choice of a multidimensional poverty index over broad classes of indices, as well as robustness over the manner in which multidimensional indicators interact in generating overall individual well-being. A further goal of the paper is to derive the sampling distribution of estimators of various useful tools for multidimensional poverty analysis, in such a way that one may infer from sample estimates the true population value of poverty measures.

\section{Multiple indicators of well-being}

\subsection{Poverty lines and poverty indices in two dimensions}

Let $x$ and $y$ be two indicators of individual well-being 2 . These could be, for instance, income, expenditures, caloric consumption, life expectancy, height, body mass, the extent of personal safety and freedom, etc. Denote the set of poverty lines for the indicators of well-being $x$ and $y$ as $z_{x}(y)$ and $z_{y}(x)$, respectively. The formulation $z_{x}(y)$ allows for the poverty line in the dimension of $x$ to depend on well-being in the dimension of $y$, and conversely for $z_{y}(x)$. This allows different dimensions of well-being to be at least partial substitutes in the attainment of an overall level of individual well-being, and thus in determining whether someone is poor or not. In such cases, we will expect that the poverty line in the $x$ dimension should not increase as $y$ increases, and vice-versa, that is, $z_{x}^{(1)}(y) \leq 0$ and $z_{y}^{(1)}(x) \leq 0$, where a superscript (1) indicates the first-order derivative of the function with respect to its argument ${ }^{3}$. We thus make this assumption from now

\footnotetext{
${ }^{1}$ Atkinson and Bourguignon $(1982,1987)$ first used this approach in the context of multidimensional social welfare.

${ }^{2}$ For expositional simplicity, we focus on the case of two dimensions of individual well-being. We will illustrate later in section 2.6 the extension to more than two dimensions.

${ }^{3}$ Note here that we implicitly assume that $z_{x}^{(1)}(y) z_{y}^{(1)}(x)$ exist everywhere. This assumption, which is made
} 
onwards.

We can think of $\left(y, z_{x}(y)\right)$ and $\left(z_{y}(x), x\right)$ as poverty frontiers along which the overall well-being of an individual is precisely equal to the poverty level of well-being, and below which individuals are in poverty. In other words, the frontier defined by $\left(y, z_{x}(y)\right)$ and $\left(z_{y}(x), x\right)$ can be interpreted as an indifference curve along which well-being precisely equals the poverty level of "utility". Within that perspective, the inverse of $z_{x}(y)$ is simply $z_{y}(x): x \equiv z_{x}\left(z_{y}(x)\right)$. Thus, if we know $z_{x}(y)$, we also know $z_{y}(x)$ by inversion, and conversely.

Assume for expositional simplicity that the joint distribution function over $x$ and $y$ is differentiable with respect to each variable, and denote the joint density function by $f(x, y)$. For analytical simplicity, we focus in this paper on classes of additive multidimensional poverty indices. An additive poverty index that combines the two dimensions of well-being can be defined generally as $P\left(z_{x}(y), z_{y}\right)$ :

$$
P\left(z_{x}(y), z_{y}\right)=\int_{0}^{z_{y}} \int_{0}^{z_{x}(y)} \pi\left(x, y ; z_{x}(y), z_{y}(x)\right) f(x, y) d x d y
$$

where $z_{y}$ is an upper limit of poverty lines in the $y$ dimension (which can tend to infinity) and where $\pi\left(x, y ; z_{x}(y), z_{y}(x)\right)$ is the contribution to poverty of an individual with wellbeing indicators $x$ and $y$, such that:

$$
\pi\left(x, y ; z_{x}(y), z_{y}(x)\right)\left\{\begin{array}{l}
\geq 0 \text { if } x \leq z_{x}(y) \text { and } y \leq z_{y}(x) \\
=0 \text { otherwise. }
\end{array}\right.
$$

For expositional convenience, we will sometimes refer to $\pi\left(x, y ; z_{x}(y), z_{y}(x)\right)$ simply as $\pi(x, y)$.

Depending on the shape of the function $z_{x}(y)$, the above formulation allows for a mixture of both an intersection and a union approach to measuring multidimensional poverty. To see why and how, consider Figure 1. If $z_{y}=c_{y}$ and $z_{x}(y)=c_{x}$, where $c_{y}$ and $c_{x}$ are constants, then (1) is an intersection poverty index: it considers someone to be in poverty only if she is poor in both of the two dimensions of $x$ and $y$, and therefore if she lies within the dashed rectangle of Figure 11. If, however, $z_{x}(y) \equiv z_{x}^{*}(y)$ in Figure 1 (the L-shaped, dotted line), with $z_{y} \rightarrow \infty$, then (1) is a union poverty index: it considers someone to be in poverty if she is poor in either of the two dimensions, i.e., if $x \leq c_{x}$ or if $y \leq c_{y}$, and therefore if she lies below or to the right of the dotted line of Figure 1 , Finally, the bi-dimensional set of poverty lines $z_{x}^{* *}(y)$ in Figure 1 provides an intermediate approach, for which the poverty line in the $x$ dimension is a decreasing function of $y$. Someone can be poor even if $y>c_{y}$, if, in the other dimension, her well-being indicator is such that $x \leq z_{x}^{* *}(y)$.

for expositional simplicity, is not strictly necessary, so long as $z_{x}(y)$ is non-increasing over $y$. This includes the possibility that $z_{x}(y)$ not be continuous and not be differentiable everywhere in $y$. 


\subsection{Poverty dominance in two dimensions}

Before making comparisons that are robust across a broad class of poverty indices, it is helpful to introduce one particular example of the multidimensional poverty indices in equation (1), a bi-dimensional extension of the FGT (Foster, Greer, and Thorbecke (1984)) index:

$$
P\left(z_{x}(y), z_{y} ; \alpha_{x}, \alpha_{y}\right)=\int_{0}^{z_{y}} \int_{0}^{z_{x}(y)}\left(z_{x}(y)-x\right)^{\alpha_{x}}\left(z_{y}(x)-y\right)^{\alpha_{y}} f(x, y) d x d y
$$

for $\alpha_{x} \geq 0$ and $\alpha_{y} \geq 0$. This index plays an important role in the robust comparisons that we introduce below. The parameters $\alpha_{x}$ and $\alpha_{y}$ capture the aversion to inequality in poverty in the $x$ and in the $y$ dimensions, respectively. $P\left(z_{x}(y), z_{y} ; 0,0\right)$ gives a bidimensional poverty headcount, namely, the proportion of individuals with both of their well-being indicators in the poverty domain defined as in Figure $1, P\left(z_{x}(y), z_{y} ; 1,0\right)$ sums the poverty gaps in $x$ (given by $\max \left(z_{y}(x)-y, 0\right)$ ) for those that are poor in $y$, and then normalizes this sum by the size of the total population. $P\left(z_{x}(y), z_{y} ; 1,1\right)$ sums the product of the poverty gaps in $x$ and in $y$, again normalized by the size of the total population, and can therefore be thought of as a bidimensional average poverty gap index, with the weights on the poverty gaps in one dimension being the poverty gaps in the other dimension. Analogous interpretations exist for other combinations of $\alpha_{x}$ and $\alpha_{y}$ values.

Instead, however, of relying on the ranking of one or a few arbitrary poverty indices and using one or a few poverty frontiers, we will investigate the ranking of poverty over areas of poverty frontiers and classes of poverty indices, classes that are defined in terms of the reactions of $\pi\left(x, y ; z_{x}(y), z_{y}(x)\right)$ to changes in $x$ and $y$. This approach will gener-

ate tests of whether multidimensional poverty rankings are robust to the index and frontier chosen, that is, tests that are valid for every one of a variety of ways of aggregating individual well-being indicators into indices of aggregate poverty over an area of poverty frontiers. These tests of multidimensional poverty robustness can be carried out at different orders of poverty dominance in each dimension of well-being.

To consider poverty dominance at a combination of two separate orders of dominance, $s_{x}$ for $x$ and $s_{y}$ for $y$, we need to define bi-dimensional stochastic dominance surfaces, denoted by $D^{s_{x}, s_{y}}\left(z_{x}, z_{y}\right)$ :

$$
D^{s_{x}, s_{y}}\left(z_{x}, z_{y}\right)=c \int_{0}^{z_{y}} \int_{0}^{z_{x}}\left(z_{x}-x\right)^{s_{x}-1}\left(z_{y}-y\right)^{s_{y}-1} f(x, y) d x d y
$$

where $c=\left(\left(s_{x}-1\right) !\left(s_{y}-1\right) !\right)^{-1}$ is a constant which plays no role in the interpretation and in the comparisons of poverty in which we are interested, and which will therefore generally be ignored. When $z_{x}(y) \equiv z_{x}$, it is clear from (3) and (4) that the bi-dimensional dominance surface, $D^{s_{x}, s_{y}}\left(z_{x}, z_{y}\right)$, is equal to the bi-dimensional FGT poverty index, $P\left(z_{x}, z_{y} ; \alpha_{x}=s_{x}-1, \alpha_{y}=s_{y}-1\right)$, times the constant $c$. Otherwise, the two differ. 
We assume that the general poverty index in (1) is left differentiable $e^{4}$ over the regions of $\left[0, z_{x}(y)\right]$ and $\left[0, z_{y}\right]$ up to the relevant orders of dominance, $s_{x}$ for derivatives with respect to $x$ and $s_{y}$ for derivatives with respect to $y$. Denote by $\pi^{x}$ the first derivative $e^{5}$ of $\pi\left(x, y ; z_{x}(y), z_{y}(x)\right)$ with respect to $x$; by $\pi^{y}$ the first derivative of $\pi\left(x, y ; z_{x}(y), z_{y}(x)\right)$ with respect to $y$; by $\pi^{x y}$ the derivative of $\left.\pi\left(x, y ; z_{x}(y), z_{y}(x)\right)\right)$ with respect to $x$ and to $y$; and treat similar expressions accordingly.

We first assume that the poverty indices over which we want to assess the robustness of poverty comparisons are continuous along the poverty frontier, namely, that $\pi\left(z_{x}(y), y\right.$; $\left.z_{x}(y), y\right)=0$ for all values of $y$. This excludes the multidimensional poverty headcount, which is discontinuous at the poverty frontier; we will come back to the case of this particular index below. We then define the following class $\ddot{\Pi}^{1,1}\left(z_{x}(y), z_{y}\right)$ of bidimensional poverty indices:

$$
\ddot{\Pi}^{1,1}\left(z_{x}(y), z_{y}\right)=\left\{\begin{array}{l|l}
P\left(z_{x}(y), z_{y}\right) & \begin{array}{l}
z_{x}^{(1)}(y) \leq 0 \\
\pi\left(z_{x}(y), y ; z_{x}(y), y\right)=0, \forall y \\
\pi^{x} \leq 0, \pi^{y} \leq 0 \text { and } \pi^{x y} \geq 0, \forall x, y .
\end{array}
\end{array}\right\}
$$

The first assumption in (5) says that the poverty frontier $z_{x}(y)$ is weakly decreasing in $y$ : this was discussed above in Section 2.1. The second assumption is the above-mentioned continuity assumption at the poverty frontier. The third assumptions say that indices that are members of $\ddot{\Pi}^{1,1}$ are weakly decreasing in $x$ and in $y$, and that they are also more affected by a change in $x$ the lower is the value of $y$. These assumptions imply that an increase in either $x$ or $y$ is good for poverty reduction, and also that the marginal poverty benefit of an increase in either $x$ or $y$ decreases with the value of the other variable. These implications would seem to be ethically unproblematic. For the indices to be nondegenerate, we must have that $\pi^{x}<0, \pi^{y}<0$ and $\pi^{x y}>0$ over some ranges of $x$ and $y$. 6

Denote by $\Delta F=F_{A}-F_{B}$ the difference between a function $F$ for $A$ and for $B$. The class of indices defined in (5) then gives rise to the following $\ddot{\Pi}^{1,1}$ bi-dimensional dominance condition:

Theorem 1 ( $\ddot{\Pi}^{1,1}$ poverty dominance)

$$
\begin{array}{r}
\Delta P\left(\zeta_{x}(y), \zeta_{y}\right)>0, \forall P\left(\zeta_{x}(y), \zeta_{y}\right) \in \ddot{\Pi}^{1,1}\left(\zeta_{x}(y), \zeta_{y}\right) \\
\text { and } \forall \zeta_{y} \in\left[0, z_{y}\right] \text { and } \forall \zeta_{x}(y) \in\left[0, z_{x}(y)\right] \\
\text { iff } \Delta D^{1,1}(x, y)>0, \forall y \in\left[0, z_{y}\right] \text { and } \forall x \in\left[0, z_{x}(y)\right] .
\end{array}
$$

\footnotetext{
${ }^{4}$ This differentiability assumption is made for expositional simplicity. It could be relaxed.

${ }^{5}$ The derivatives include the effects of $y$ in the term $z_{x}(y)$ and of $x$ in the term $z_{y}(x)$.

${ }^{6}$ Note also that the inequalities in (5) are weak, which is different from the strong inequalities that are often found in the literature. This is consistent, however, with the way in which we will proceed to test dominance - we will test for strict ordering of the dominance surfaces, instead of the weak orderings often tested in the empirical literature.
} 
Proof: See the appendix.

If condition (6) is met, Theorem 1 says that poverty will be unambiguously higher in $A$ than in $B$ for all of the poverty indices that are members of $\ddot{\Pi}^{1,1}$ and for all poverty frontiers for which $\zeta_{y} \in\left[0, z_{y}\right]$ and $\zeta_{x}(y) \in\left[0, z_{x}(y)\right]$. Because of its generality, this is a powerful ordering of multidimensional poverty across $A$ and $B$.

Condition (6) requires that the dominance surface $D^{1,1}(x, y)$ be higher for $A$ than for $B$ for all pairs of intersection poverty frontiers, $(x, y)$, for which $x \in\left[0, z_{x}(y)\right]$ and $y \in\left[0, z_{y}\right]$. To see more clearly what this implies, return to Figure 1 and consider first an intersection poverty definition. In this case, $z_{y}$ and $z_{x}(y)$ are upper bounds that can be set to the constants $c_{y}$ and $c_{x}$ respectively, and the relevant domain for the test is the rectangle defined by the axes and the upper bounds $\left(c_{x}, c_{y}\right)$. Thus, to establish a robust poverty comparison on this domain, we must check that $A$ 's dominance surface is above $B$ 's at every point over this rectangle. Note, however, that once this is established, we are assured of a robust poverty ordering not only at the precise intersection poverty frontier, $\left(c_{x}, c_{y}\right)$, but also for all other poverty frontiers $\left(y, z_{x}(y)\right)$ of bi-dimensional poverty lines which "fit" into the rectangle. These alternative poverty frontiers would include all of the intermediate frontiers (of the type of $z_{x}^{* *}(y)$ in Figure 1) that could fit in the rectangle defined by $(0,0)$ and $\left(c_{x}, c_{y}\right)$.

For a union estimator, the test domain of these poverty frontiers is the L-shaped region defined in Figure 1 by $x \in\left[0, z_{x}^{*}(y)\right]$ and $y \in[0, \infty]$. Again, condition (6) requires that the dominance surface be higher for $A$ than for $B$ for all pairs of intersection poverty frontiers over that region. If that is established, we are assured of a robust poverty ordering for all other union, intersection, or intermediate poverty frontiers $\left(y, z_{x}(y)\right)$ which are included in that testing area. The extension to more general functions such as $z_{x}^{* *}(y)$ follows naturally.

Theorem 1 is convenient because it does not require comparing all of the (infinitely numbered) possible poverty indices that are members of $\ddot{\Pi}^{1,1}$, just the dominance surfaces. In addition, it does not require that we test poverty dominance for all possible frontiers $\left(y, z_{x}(y)\right)$, but just for the intersection frontiers that fit within the domain of the test. A cost of this simplification is that $\ddot{\Pi}^{1,1}$ poverty dominance does not guarantee that indices that are discontinuous at the poverty frontier will be greater in $A$ than in $B$ for all poverty frontiers that belong to the test domain, since these discontinuous indices are not members of $\ddot{\Pi}^{1,1}$. We will come back to the continuity issue in Section 4

\subsection{Higher order dominance tests}

For higher-order dominance, we either increase the order in one dimension or in both simultaneously. Either approach adds further assumptions on the effects of changes in either $x$ or $y$ on aggregate poverty, and thus limits the applicable class of poverty measures. These further assumptions are analogous to those found in the unidimensional dominance literature, and impose that indices react increasingly favorably to increases in living standards at the bottom of the distribution of well-being. The assumptions further require that 
the reactions of the indices to changes in one indicator be the greater the lower the level of the other indicator of well-being.

To illustrate this, assume in addition to the above conditions for $\ddot{\Pi}^{1,1}$ that the firstorder derivative $\pi^{x}(x, y)$ is continuous at $x=z_{x}(y)$, that is, that $\pi^{x}\left(z_{x}(y), y\right)=0$. Further suppose that equalizing transfers in $x$ at a given value of $y$ weakly reduce aggregate poverty, and that this effect is decreasing in the value of $y$. We then obtain the following class of bidimensional poverty indices:

$$
\ddot{\Pi}^{2,1}\left(z_{x}(y), z_{y}\right)=\left\{\begin{array}{l|l}
P\left(z_{x}(y), z_{y}\right) & \begin{array}{l}
P\left(z_{x}(y), z_{y}\right) \in \ddot{\Pi}^{1,1}\left(z_{x}(y), z_{y}\right) \\
\pi^{x}\left(z_{x}(y), y\right)=0 \forall y \\
\pi^{x x}(x, y) \geq 0 \forall x \\
\text { and } \pi^{x x y}(x, y) \leq 0, \forall x, y .
\end{array}
\end{array}\right\}
$$

This leads to the following dominance condition:

\section{Theorem $2\left(\ddot{\Pi}^{2,1}\right.$ poverty dominance $)$}

$$
\begin{array}{r}
\Delta P\left(\zeta_{x}(y), \zeta_{y}\right)>0, \forall P\left(\zeta_{x}(y), \zeta_{y}\right) \in \ddot{\Pi}^{2,1}\left(\zeta_{x}(y), \zeta_{y}\right) \\
\text { and } \forall \zeta_{y} \in\left[0, z_{y}\right] \text { and } \forall \zeta_{x}(y) \in\left[0, z_{x}(y)\right] \\
\text { iff } \Delta D^{2,1}(x, y)>0, \forall y \in\left[0, z_{y}\right] \text { and } \forall x \in\left[0, z_{x}(y)\right] .
\end{array}
$$

Proof: See appendix.

This tests simultaneous dominance of order 2 for $x$ and of order 1 for $y$ by checking whether the average poverty gap in $x$ (given by $D^{2,1}(x, y)$ ), progressively cumulated in the dimension of $y$, is greater in $A$ than in $B$, regardless of which intersection poverty frontier, $(x, y)$ with $x \in\left[0, z_{x}\left(\zeta_{y}\right)\right]$ and $y \in\left[0, z_{y}\right]$, is chosen. The ordering properties are analogous to those of Theorem 1 .

Although it may not prove necessary, we can move to higher orders of dominance in the $x$ dimension. The classes of poverty indices belonging to $\ddot{\Pi}^{s_{x}, 1}\left(z_{x}(y), z_{y}\right)$ become increasingly restricted as $s_{x}$ increases. For $\ddot{\Pi}^{3,1}\left(z_{x}(y), z_{y}\right)$ for instance, poverty indices must obey the principle of transfer sensitivity ${ }^{7}$ in $x$, and react more to a favorable composite transfer the lower the value of $y$. Higher values of $s_{x}$ imply compliance with higher-order principles of transfers $\frac{8}{\text {. }}$

In addition, we can simultaneously increase both $s_{x}$ and $s_{y}$. The procedures, classes of poverty indices, and dominance relationships are analogous to those described above. For instance, the conditions for membership in $\ddot{\Pi}^{2,2}\left(z_{x}(y), z_{y}\right)$ require that the poverty indices be convex in both $x$ and $y$, and that they therefore obey the principle of transfers in both of these dimensions. They also require that this principle be stronger in one dimension of well-being the lower the level of the other dimension of well-being. Finally, they also impose that the level of convexity in one dimension of well-being be convex

${ }^{7}$ For a definition, see for instance Shorrocks and Foster (1987).

${ }^{8}$ For an illustration of this in the unidimensional setting, see Fishburn and Willig (1984). 
in the level of the other indicator of well-being. The dominance condition then checks whether $D^{2,2}(x, y)$ is greater in $A$ than in $B$ for all combinations of poverty lines for which $x \in\left[0, z_{x}(y)\right]$ and $y \in\left[0, z_{y}\right]$.

\subsection{Relevance of the methods}

The methods that we propose above are more general than two other methods that researchers have used to consider poverty in multiple dimensions. One approach has been to combine many indicators of well-being into one, unidimensional index, and then compare that index across populations. The best-known example is the Human Development Index (UNDP, 1990). To see how this method differs from ours, return to Figure 1, which shows the domain for tests using two well-being variables. If we chose to compare a single aggregate welfare index of these two variables, the effect would essentially be to reduce the domain for the test to a single line emanating from the origin and being closer to the $\mathrm{x}$ or $\mathrm{y}$ axis according to the weight that $\mathrm{x}$ and $\mathrm{y}$ receive in the welfare index. Our proposed tests clearly generalize on this approach.

The second approach is to compare many indicators of well-being independently. Such univariate comparisons are also a special case of our approach. To see this, recall that the dominance surface $D^{s_{x}, s_{y}}(x, y)$ is cumulative in both dimensions. Hence, integrating out one dimension only leaves the univariate dominance curve for the other dimension of well-being. In terms of Figure 1, the domain of separate univariate tests would be a horizontal line up to $c_{y}$ at $x=\infty$ for the $y$ variable, and a vertical line up to $c_{x}$ at $y=\infty$ for the $x$ variable.

There are then two ways in which our test could differ from this "one-at-a-time" approach. First, it is possible that the univariate dominance curve for $A$ lies above that for $B$ at both $x=\infty$ and $y=\infty$ for the relevant range of poverty lines, but that $A$ is not above $B$ at one or more interior points in the test domain shown in Figure 1. In this case, the one-at-a-time approach would conclude that poverty is higher in $A$ than $B$, but our bivariate approach would not. Indeed, the bivariate approach checks the joint distribution of all indicators of well-being, and it is thus able to show the correlation across such indicators, which is of ethical importance since it helps capture "multiple" poverty - that is, the joint incidence of deprivation in multiple dimensions. One-at-a-time analysis fails to do this. Alternatively, it is possible for the dominance surfaces to cross at $x=\infty$ and/or $y=\infty$, but for $A$ 's surface to be above $B$ 's for a large area of interior points in the test domain. In this case, the one-at-a-time approach would not be able to establish a ranking of poverty, but our test would for an intersection definition of poverty and some intermediate definitions. 9

To visualize these methodological differences, consider Figure 2, which graphs a typical dominance surface. A larger "hump" in the middle of the surface corresponds to a

${ }^{9}$ A union definition, however, would include the margins of the surface in its test domain and would therefore not find a robust ordering of poverty. 
larger positive correlation between the two well-being variables. Also, the univariate dominance curve for one dimension is found at the upper extreme of the dominance surface's other dimension. On Figure 2, the univariate curve for the log of household expenditures lies on the extreme right of the surface, while that for the height-for-age $z$-score (to which we return below) is behind it.

When we make dominance comparisons, we test for the difference between two surfaces like the one shown in Figure 2. Figure 3 depicts such a difference for the case in which one surface has highly correlated welfare variables while the second does not (the difference in these surfaces "bulges" in the middle). Although differences in the univariate dominance curves in both dimensions clearly cross the origin (at the extreme left and right of the figure), there is a significant interior section where the first surface is entirely above the second. Hence, there are poverty frontiers for which the first distribution has more poverty than the second. Conversely, we could think of shifting Figure 3 down such that the univariate differences were all negative. The first distribution would then dominate the second in both dimensions individually, but there would still be a section in the middle where the first surface would lie above the second. Thus, there would be no bivariate poverty dominance due to the first distribution showing two much incidence of multiple deprivation. We will give further examples of this in section 2.6

\subsection{Estimation and inference}

We now consider the estimation of the tools derived above for multidimensional poverty analysis. In this, we generalize to more than one dimension some of the results of Davidson and Duclos (2000).

Suppose first that we have a random sample of $N$ independently and identically distributed observations drawn from the joint distribution of $x$ and $y$. We can write these observations of $x^{L}$ and $y^{L}$, drawn from a population $L$, as $\left(x_{i}^{L}, y_{i}^{L}\right), i=1, \ldots, N$. A natural estimator of the dominance surfaces $D^{s_{x}, s_{y}}\left(z_{x}, z_{y}\right)$ (see 4 ) is then:

$$
\begin{aligned}
& \hat{D}_{L}^{s_{x}, s_{y}}\left(z_{x}, z_{y}\right) \\
& =\int_{0}^{z_{y}} \int_{0}^{z_{x}}\left(z_{y}-y\right)^{s_{y}-1}\left(z_{x}-x\right)^{s_{x}-1} d \hat{F}_{L}(x, y) \\
& =\frac{1}{N} \sum_{i=1}^{N}\left(z_{y}-y_{i}^{L}\right)^{s_{y}-1}\left(z_{x}-x_{i}^{L}\right)^{s_{x}-1} I\left(y_{i}^{L} \leq z_{y}\right) I\left(x_{i}^{L} \leq z_{x}\right) \\
& =\frac{1}{N} \sum_{i=1}^{N}\left(z_{y}-y_{i}^{L}\right)_{+}^{s_{y}-1}\left(z_{x}-x_{i}^{L}\right)_{+}^{s_{x}-1}
\end{aligned}
$$

where $\hat{F}$ denotes the empirical joint distribution function, $I(\cdot)$ is an indicator function equal to 1 when its argument is true and 0 otherwise, and $f_{+}(x)=\max (0, x)$. A more general case of (9) is that of the multidimensional FGT and other additive indices with an arbitrarily-defined poverty frontier, a case which we consider in the proof of Theorem 3 in the Appendix. For arbitrary $s_{x}$ and $s_{y}$, (9) has the convenient property of being a simple sum of IID variables, even allowing for the fact that $x$ and $y$ and will generally be correlated across observations. 
The following theorem allows us to perform statistical inference in the case in which we have a sample from each of two populations, $A$ and $B$, that may or may not have been drawn independently from each other.

Theorem 3 Let the joint population moments of order 2 of $\left(z_{y}-y^{A}\right)_{+}^{s_{y}-1}\left(z_{x}-x^{A}\right)_{+}^{s_{x}-1}$ and $\left(z_{y}-y^{B}\right)_{+}^{s_{y}-1}\left(z_{x}-x^{B}\right)_{+}^{s_{x}-1}$ be finite. Then $N^{1 / 2}\left(\hat{D}_{A}^{s_{x}, s_{y}}\left(z_{x}, z_{y}\right)-D_{A}^{s_{x}, s_{y}}\left(z_{x}, z_{y}\right)\right)$ and $N^{1 / 2}\left(\hat{D}_{B}^{s_{x}, s_{y}}\left(z_{x}, z_{y}\right)-D_{B}^{s_{x}, s_{y}}\left(z_{x}, z_{y}\right)\right)$ are asymptotically normal with mean zero, with asymptotic covariance structure given by $(L, M=A, B)$ :

$$
\begin{aligned}
& \lim _{N \rightarrow \infty} N \operatorname{cov}\left(\hat{D}_{L}^{s_{x}, s_{y}}\left(z_{x}, z_{y}\right), \hat{D}_{M}^{s_{x}, s_{y}}\left(z_{x}, z_{y}\right)\right) \\
& =c^{2} E\left(\left(z_{y}-y^{L}\right)_{+}^{s_{y}-1}\left(z_{x}-x^{L}\right)_{+}^{s_{x}-1}\left(z_{y}-y^{M}\right)_{+}^{s_{y}-1}\left(z_{x}-x^{M}\right)_{+}^{s_{x}-1}\right) . \\
& \quad-D_{L}^{s_{x}, s_{y}}\left(z_{x}, z_{y}\right) D_{M}^{s_{x}, s_{y}}\left(z_{x}, z_{y}\right)
\end{aligned}
$$

Proof: See the appendix.

When the samples from the populations $A$ and $B$ are independent, the variance of each of $\hat{D}_{A}^{s_{x}, s_{y}}\left(z_{x}, z_{y}\right)$ and $\hat{D}_{B}^{s_{x}, s_{y}}\left(z_{x}, z_{y}\right)$ can be found by using (10) and by replacing $N$ by $N_{A}$ and $N_{B}$ respectively. The covariance between the two estimators is then zero. The elements of the asymptotic covariance matrix can be estimated consistently using their sample equivalents.

\subsection{Examples}

As a first example, consider the question: are rural people poorer than the urban ones in Viet Nam? Many studies, of Viet Nam and elsewhere, find that people living in rural areas tend to be poorer when judged by expenditures or income alone. However, it is possible that people are better nourished in rural than urban areas, ceteris paribus, because they have tastes for foods that provide nutrients at a lower cost, or because unit prices of comparable food commodities are lower. In such cases, including an indicator of nutritional status may change the relative well-being of rural and urban residents. To test this, we measure welfare in two dimensions: per capita household expenditures and nutritional status, as measured by a childs' gender and age standardized height, transformed into standard deviation or z-scores. Stunted growth in children is widely used as an indicator of malnutrition and poor health. The sample comes from the Viet Nam Living Standards Measurement Survey carried out in 199310. This is a nationally representative household survey that collected detailed expenditure and anthropometric data. The latter, however, are available only for children younger than 60 months, so our sample is actually these children only, rather than for all of the members of the households interviewed.

\footnotetext{
${ }^{10}$ Information on the LSMS surveys is available in Grosh and Glewwe (1998). Information about the specific survey that we use is available at the LSMS website: http://www.worldbank.org/html/prdph/lsms/country/vn93/vn93bid.pdf.
} 
The test described in equation (6) requires comparison of the two dominance surfaces of urban and rural children in Viet Nam: this is shown in Figure 4 for $s_{x}=s_{y}=1$. The $y$ axis measures the height-for-age $z$-score (stunting); the $x$ axis measures the per capita expenditures for the child's household; and the $z$ axis measures the cumulative proportion of children that fall below the points defined in the $(x, y)$ domain. The poorest children are in the front left-hand corner of the graph. If the rural dominance surface is above the urban surface over the relevant area of poverty frontiers (values of per capita expenditures and stunting), poverty is higher (more people are below the given well-being levels in each dimension) in rural areas. This conclusion is then robust to the choice of poverty indices in the class $\ddot{\Pi}^{1,1}\left(z_{x}(y), z_{y}\right)$, and robust to the choices of poverty frontiers within $\left[0, z_{y}\right]$ and $\left[0, z_{x}(y)\right]$.

In theory, we should test over the entire area defined by $z_{x}(y)$ and $z_{y}$, but it is more practical to choose a grid of points in the $(x, y)$ domain over which to test. Here we use a grid that is 20x20, spread evenly over the entire domain of the log of per capita expenditures and the height-for-age $z$-score. Following Howes (1996), we test for a significant difference in the dominance surface at each point of the grid, and reject the null of nondominance of $A$ by $B$ only if all of the $t$-statistics have the right sign and are significantly different from 0 .

Figure 4 indicates clearly that, over almost the entire range of expenditures and stunting, rural children are poorer than urban. Table 1 shows whether these statements are statistically significantly at the 5\% level. A negative sign indicates that the urban dominance surface is significantly below the rural one, a positive sign indicates the opposite, and a zero indicates that the difference is not statistically significant. The negative differences are statistically significant for any reasonable pair of poverty lines (except at the very bottom right of Table 1 . Hence, by Theorem 1 , the conclusion that rural children are poorer than urban ones is valid for almost any intersection, union or intermediate poverty frontier.

Our second example tests for first-order poverty dominance in three dimensions. We ask whether poverty declined in Ghana between 1993 and 1998, using data from the Demographic and Health Surveys. The three welfare variables that we consider are for children under five years old: their survival probability, their height-for-age z-score (stunting), and an index of their household's assets. ${ }^{11}$ We compare dominance surfaces for these three measures in 1993 and 1998, the two years for which DHS data exist. While we cannot graph the resulting four-dimensional surface, Figure 5 summarizes the results of the

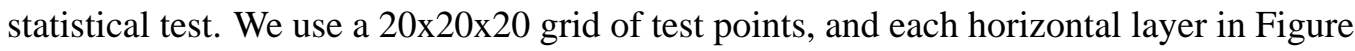
5 is similar to Table 1 in the previous example.12 A light gray point indicates that the 1998 surface is significantly above the 1993 surface; a darker gray point indicates that the 1998 surface is significantly below the 1993 surface; and a black point indicates that they are statistically indistinguishable at the five-percent significance level. It is clear from the

\footnotetext{
${ }^{11}$ Information on the estimation of survival probabilities and the asset index may be found in Sahn, Stifel, and Younger (1999) and Sahn and Stifel (2000).

${ }^{12}$ We have excluded some of the horizontal layers to make the graph more legible.
} 
figure that there is no robust poverty dominance result. Over some of the domain, poverty does seem to have declined between 1993 and 1998. But in significant areas, particularly for low values in the asset dimension, the reverse is true.

In addition to showing that our tests are possible in more than two dimensions, this example shows the importance of checking for the robustness of poverty comparisons using tests such as those we employ. For the intersection headcount, shown by points on the dominance surfaces, a judicious choice of the poverty lines could lead one to conclude that poverty worsened, improved, or did not change, depending on the specific choice. None of these results would be robust, but any would seem plausible if it appeared on its own.

The next two examples highlight the difference between using bivariate dominance tests $v s$. one-at-a-time univariate tests on the same variables. Table 2 gives the results for tests of the differences in the dominance surfaces for stunting and child survival probability in Cameroon and Madagascar. The data come from the 1997 Demographic Health Surveys (DHS) in those countries. ${ }^{13}$ The "one-at-a-time" dominance curves are given in the last row of the table (for survival probability) and in the last column (for stunting). It is clear that these univariate comparisons would conclude that poverty is worse in Madagascar than in Cameroon, whether measured by stunting or survival probability. Nevertheless, the bivariate comparison shows several internal points where the surfaces are not significantly different, including two where the point estimate of the difference is in fact positive. So our method would not come to the same conclusion, finding instead that there is no statistically-significant first-order poverty ordering of these two populations.

Table 3 shows the other possibility for different conclusions. These results are also for tests of the differences between first-order dominance surfaces for stunting and child survival probability, in Colombia and the Dominican Republic, and come from the DHS surveys for those countries, carried out in 1995 and 1996 respectively. In this case, there is dominance on one margin (for survival probability), but not the other (stunting), so the one-at-a-time approach would not find poverty to be necessarily lower in one population than the other. However, Colombia's dominance surface is significantly below the Dominican Republic's over a very large range of the interior points, suggesting that under an intersection definition of poverty, and several intermediate ones as well, poverty was robustly lower in Colombia than in the Dominican Republic.

\section{Bounds to multidimensional dominance}

Implementing the approaches to multidimensional poverty dominance developed in section 2 requires specifying the position of the upper frontier $z_{x}(y)$ and $z_{y}$. Although there may be some intuitive feel that extreme values of $z_{x}(y)$ and $z_{y}$ are not sensible, there

\footnotetext{
${ }^{13}$ Information on these surveys is available at http://www.measuredhs.com. The child survival probability is estimated as in Sahn, Stifel, and Younger (1999).
} 
is rarely reliable empirical evidence about what the precise value of these upper bounds should be. Specifying their value a priori is thus necessarily subject to some degree of arbitrariness. An alternative approach that gets around such arbitrariness is to estimate directly from the samples the upper limit of the poverty frontiers for which multidimensional poverty dominance holds in the sample. These upper bounds can be termed critical poverty frontiers since they will determine the area of poverty frontiers which may not be exceeded for a robust multidimensional ordering of poverty to be possible. The researcher can then judge whether these limits are sufficiently high to justify a conclusion of poverty dominance.

To develop this idea further, assume that these critical poverty frontiers exist in the two populations of multidimensional well-being being compared. Assume therefore that $B$ initially dominates $A$ but that their dominance surfaces eventually cross and that the ranking of the dominance surfaces is thus eventually reversed. Hence, for a given value of $y$, let $\zeta_{x}^{+}(y)$ then be the first crossing point ${ }^{14}$ of the surfaces in the $x$ dimension, with $D_{A}^{s_{x}, s_{y}}\left(\zeta_{x}^{+}(y), y\right)=D_{B}^{s_{x}, s_{y}}\left(\zeta_{x}^{+}(y), y\right)$. Carrying out this exercise for a range $\left[0, z_{y}\right]$ of $y$ leads to the estimation of a critical poverty frontier in the $x$ dimension. By the results derived in section 2 , this procedure will provide an estimate of the space $\left[0, z_{x}(y)\right]$ and $\left[0, z_{y}\right]$ in which we can locate all of the possible poverty frontiers (union, intersection, or intermediate) for which there is necessarily more poverty in $A$ than in $B$ for all poverty indices that are members of $\ddot{\Pi}^{s_{x}, s_{y}}$. This procedure can be applied for any desired orders of bi-dimensional dominance $s_{x}$ and $s_{y}$, and can be generalized to more dimensions.

To summarize, the critical poverty frontier $\zeta_{x}^{+}(y)$ limits the poverty frontiers for which poverty in $A$ can be said to be robustly above that in $B$. For poverty frontiers lying above $\zeta_{x}^{+}(y)$, it would always be possible to find a poverty index within $\ddot{\Pi}^{s_{x}, s_{y}}$ that would show more poverty in $B$ than in $A^{15}$. The frontier $\zeta_{x}^{+}(y)$ also locates the intersection poverty frontiers for which the bi-dimensional FGT poverty indices would be exactly the same in the two distributions. In other words, a social-decision maker using the multidimensional intersection FGT index as a social evaluation function would be indifferent between the two distributions at any pair of poverty lines along that frontier.

\subsection{Estimation and inference}

To establish the sampling distribution of estimators of the critical frontier $\zeta_{x}^{+}(y)$, assume that within some bottom area $x \in\left[0, c_{x}\right]$ and at a given value of $y$, the population dominance surface for $A$ lies above that for $B$, but that these surfaces cross (exactly) in the population at some higher critical point $\zeta_{x}^{+}(y)$. For a fixed value of $y$, a natural estimator $\hat{\zeta}_{x}^{+}(y)$ of the location of that point can be defined by the first point above $y$ at which the sample ordering of the dominance surface changes. If the sample dominance surface for

\footnotetext{
${ }^{14}$ Note that $\zeta_{x}^{+}(y)$ will depend on the orders of dominance $\left(s_{x}, s_{y}\right)$ considered, and should formally be written as $\left.\zeta_{x}^{+}\left(y ; s_{x}, s_{y}\right)\right)$. For expositional simplicity, however, we do not make this dependence explicit.

${ }^{15}$ This is by the necessity part of conditions $(6)$ and $(8)$.
} 
$A$ were to lie always above that for $B$ above $y$, then we could set $\hat{\zeta}_{x}^{+}(y)$ to an arbitrarily large value (denote it by $z_{x}^{+}$). Formally, $\hat{\zeta}_{x}^{+}(y)$ is then defined as ${ }^{16}$ :

$$
\hat{\zeta}_{x}^{+}(y)=\sup \left\{x \mid \Delta \hat{P}\left(x, y ; s_{x}-1, s_{y}-1\right) \geq 0 \text { and } x \leq z_{x}^{+}\right\}
$$

Applying this estimator over a range of $y$ leads to an estimator of the critical frontier $\zeta_{x}^{+}(y)$. Under these conditions, the sampling distribution of $\hat{\zeta}_{x}^{+}(y)$ is given by the following theorem 4. For this, however, it is expositionally convenient to define an FGT index with a negative $\alpha_{x}$ as:

$$
\begin{aligned}
P\left(z_{x}, z_{y} ;-1, \alpha_{y}\right) & =\int_{0}^{z_{y}}\left(z_{y}-y\right)^{\alpha_{y}} f\left(y \mid x=z_{x}\right) d y f_{x}\left(z_{x}\right) \\
& =E\left[\left(z_{y}-y\right)_{+}^{\alpha_{y}} \mid x=z_{x}\right] f_{x}\left(z_{x}\right)
\end{aligned}
$$

where $f_{x}\left(z_{x}\right)$ is the marginal density of $x$ at $z_{x}$ and $f(y \mid x)$ is the conditional density of $y$ at $x$. This leads to the following theorem.

Theorem 4 Let the joint population moments of order 2 of $\left(x^{A}\right)^{\left(s_{x}-1\right)}\left(y^{A}\right)^{\left(s_{y}-1\right)}$ and $\left(x^{B}\right)^{\left(s_{x}-1\right)}\left(y^{B}\right)^{\left(s_{y}-1\right)}$ exist. If the samples from $A$ and $B$ are independent, assume that the ratio $r=N_{A} / N_{B}$ of their respective sample size tends to a constant as $N_{A}$ and $N_{B}$ tend to infinity. Under the conditions mentioned above (in particular, that $\zeta_{x}^{+}(y)$ exists in the population), $N^{1 / 2}\left(\hat{\zeta}_{x}^{+}(y)-\zeta_{x}^{+}(y)\right)$ is then asymptotically normal with mean zero, and its asymptotic variance is given by

$$
\begin{array}{ll} 
& \lim _{N \rightarrow \infty} \operatorname{var}\left(N^{1 / 2}\left(\hat{\zeta}_{x}^{+}(y)-\zeta_{x}^{+}(y)\right)\right)= \\
& {\left[\delta \cdot\left(P_{A}\left(\zeta_{x}^{+}(y), y ; s_{x}-2, s_{y}-1\right)-P_{B}\left(\zeta_{x}^{+}(y), y ; s_{x}-2, s_{y}-1\right)\right)\right]^{-2}} \\
\times \quad & {\left[\operatorname{var}\left(\left(y-y^{A}\right)_{+}^{s_{y}-1}\left(\zeta_{x}^{+}(y)-x^{A}\right)_{+}^{s_{x}-1}\right)+\operatorname{var}\left(\left(y-y^{B}\right)_{+}^{s_{y}-1}\left(\zeta_{x}^{+}(y)-x^{B}\right)_{+}^{s_{x}-1}\right)\right.} \\
& \left.-2 \operatorname{cov}\left(\left(y-y^{A}\right)_{+}^{s_{y}-1}\left(\zeta_{x}^{+}(y)-x^{A}\right)_{+}^{s_{x}-1},\left(y-y^{B}\right)_{+}^{s_{y}-1}\left(\zeta_{x}^{+}(y)-x^{B}\right)_{+}^{s_{x}-1}\right)\right]
\end{array}
$$

when the samples are dependent, and by

$$
\begin{aligned}
& \lim _{N_{A} \rightarrow \infty} \operatorname{var}\left(N_{A}^{1 / 2}\left(\hat{\zeta}_{x}^{+}(y)-\zeta_{x}^{+}(y)\right)\right)= \\
& {\left[\delta \cdot\left(P_{A}\left(\zeta_{x}^{+}(y), y ; s_{x}-2, s_{y}-1\right)-P_{B}\left(\zeta_{x}^{+}(y), y ; s_{x}-2, s_{y}-1\right)\right)\right]^{-2} } \\
\times \quad & {\left[\operatorname{var}\left(\left(y-y^{A}\right)_{+}^{s_{y}-1}\left(\zeta_{x}^{+}(y)-x^{A}\right)_{+}^{s_{x}-1}\right)+r \operatorname{var}\left(\left(y-y^{B}\right)_{+}^{s_{y}-1}\left(\zeta_{x}^{+}(y)-x^{B}\right)_{+}^{s_{x}-1}\right)\right] }
\end{aligned}
$$

when the samples are independent, and by setting $\delta=s_{x}-1$ when $s_{x}>1$, and $\delta=1$ when $s_{x}=1$.

Proof: See appendix.

\footnotetext{
${ }^{16}$ Recall that $\Delta P=P_{A}-P_{B}$.
} 


\subsection{Example}

Figure 6 shows the critical poverty frontier for which poverty, measured by children's weight-for-height $z$-score and their survival probability, is lower in Madagascar than in Egypt. A lower frontier is also drawn 2 standard errors below the estimated one. The frontier and the standard errors are calculated in the vertical dimension (weight-for-age $z$-score). The data come from the DHS surveys for these countries in 1997 and 1992, respectively. Madagascar's dominance surface is significantly below Egypt's for a large range of interior points, including all negative $z$-scores. But the bound drops sharply at higher values of the survival probability, limiting considerably the range over which union poverty dominance can be established.

\section{Multivariate dominance with discrete indicators of well-being}

We now show in this section how the results of section 2 specialize to the case of discrete distributions. This discrete setting will also help understand better the assumptions made earlier and the testing procedures involved. Suppose that the population can be split into $K$ exhaustive and exclusive population subgroups, whose population share is denoted by

$\phi(k), k=1, \ldots, K$. Hence, $\sum_{k=1}^{K} \phi(k)=1$. We can define these subgroups based on a discrete welfare measure such as literacy, political enfranchisement, access to a public service, or physical capabilities. Alternatively, we can differentiate households by their relative needs, based on size and composition, type of activities, or area of residence. In either case, the important point is that these discrete differences in the characteristics of households or individuals suggest that, for a given value of the continuous measure(s) of well-being, certain groups have lower overall well-being than others. This can be because the discrete variable is itself a measure of well-being (being illiterate is worse than being literate), or because it indicates differences in needs, prices, or poverty lines. In addition, we can suppose that there is some uncertainty as to the precise value of these differences. We will assume below that the $K$ subgroups can be ordered in decreasing value of "needs", in such a way that at common alternative indicators of well-being, individuals in subgroup 1 have more needs than individuals in subgroup 2, who have more needs than individuals in subgroup 3, and so on.

Since the relevant indices are again assumed to be additive, poverty in each of the population subgroups can be defined as:

$$
P(k ; z(k))=\int_{0}^{z(k)} \pi_{k}(x) f(x ; k) d x
$$

where $f(x ; k)$ is subgroup $k$ 's density of living standards at $x$, and $z(k)$ is subgroup $k$ 's poverty line in the dimension of $x . \pi_{k}(x ; z(k))$ is the contribution to subgroup $k$ 's poverty of an individual in that subgroup with living standard equal to $x$. Since the non-poor do 
not, by definition, contribute to total poverty, we have that $\pi_{k}(x)=0$ if $x>z(k)$. Total poverty in the population is given by:

$$
\begin{aligned}
P(z(1), \ldots, z(K)) & =\sum_{k=1}^{K} \phi(k) \int_{0}^{z(k)} \pi_{k}(x) f(x ; k) d x \\
& =\sum_{k=1}^{K} \phi(k) P(k ; z(k)) .
\end{aligned}
$$

For expositional simplicity, we will sometimes denote $P(z(1), \ldots, z(K))$ simply by $P$. One such poverty index is the sum of FGT indices across subgroups, each with its own poverty line. Denote the FGT index for subgroup $k$ by

$$
P(k ; z(k) ; \alpha)=\int_{0}^{z(k)}(z(k)-x)^{\alpha} f(x ; k) d x .
$$

The $P(k ; z(k) ; \alpha)$ indices are again closely related to the unidimensional dominance curves for subgroup $k, D^{s}(k ; z(k))$, which are defined as $c \cdot \phi(k) \cdot P(k ; z ; \alpha=s-1)$, where $c=1 /(s-1)$ ! is a constant. Total poverty as measured by the FGT index is then:

$$
P(z(1), \ldots, z(K) ; \alpha)=\sum_{k=1}^{K} \phi(k) P(k ; z(k) ; \alpha) .
$$

Note that $P(z(1), \ldots, z(K) ; 0)$ is the population headcount, with each subgroup $k$ being assigned its specific poverty line $z(k)$. Similarly, $P(z(1), \ldots, z(K) ; 1)$ is the average poverty gap in the population, again with each subgroup $k$ being assigned its specific poverty line $z(k)$. Other multidimensional additive poverty indices can be defined along similar lines, extending, for instance, the unidimensional Watts (1968) or Chakravarty (1983) poverty indices.

We assume that the poverty lines of the subgroups can be ordered from the highestneeds to the lowest-needs group as follows:

$$
z(1) \geq z(2) \geq \ldots \geq z(K) .
$$

This is the discrete analogue of condition $z_{x}^{(1)}(x) \leq 0$ in Section 2. The ordering in (20) is sensible since we assume that individuals in group $k$ have lower welfare or greater needs than those in group $k+1$, all else equal. We also suppose for analytical simplicity that $\pi_{k}(x)$ is left differentiable between 0 and $z(k)$ up to the relevant order of dominance, $s$. For first-order dominance, we then need an ordering of the first-order derivatives of $\pi_{k}(x)$ with respect to $x$, which we denote as $\pi_{k}^{(1)}$ :

$$
\pi_{1}^{(1)}(x) \leq \pi_{2}^{(1)}(x) \leq \ldots \leq \pi_{K}^{(1)}(x) \leq 0, \forall x .
$$

Assumption (21) says that an increase in $x$ causes a greater poverty reduction the greater the needs of individuals. It is the analogue of $\pi^{x y} \geq 0$ in section 2 . 


\subsection{Continuous poverty indices}

For expositional and analytical convenience, assume that the derivatives of the functions $\pi_{k}^{(1)}(x)$ are continuous at the poverty line, up to the $(s-1)^{\text {th }}$ order. For first-order dominance, this requires that:

$$
\pi_{k}(z(k))=0, \forall k=1, \ldots, K .
$$

As in the case of continuous welfare variables, note that the continuity assumption (22) is not obeyed by the multidimensional poverty headcount. The consequences of this will be discussed shortly. The class, $\Pi^{1}(z(1), \ldots, z(K))$, of multidimensional first-order poverty indices then includes all of the $P$ indices defined in equation (17) that satisfy assumptions (20), (21) and (22). This definition then leads to the following equivalence:

\section{Theorem 5 (First-order poverty dominance for heterogeneous populations)}

$$
\begin{gathered}
\Delta P(\zeta(1), \ldots, \zeta(K))>0 \\
\forall P(\zeta(1), \ldots, \zeta(K)) \in \Pi^{1}(\zeta(1), \ldots, \zeta(K)) \\
\text { and } \forall \zeta(k) \in[0, z(k)], k=1, \ldots, K \\
\text { iff } \sum_{k=1}^{i} \Delta D^{1}(k ; \zeta)>0, \forall \zeta \in[0, z(i)] \text { and } \forall i=1, \ldots, K .
\end{gathered}
$$

Proof: See Atkinson (1991) and Jenkins and Lambert (1993). For ease of reference, a proof is also shown in the appendix.

Recall that $D^{1}(k ; \zeta)$ is the headcount in subgroup $k$ for a poverty line $\zeta$, times the population share of subgroup $k . \sum_{k=1}^{i} D^{1}(k ; \zeta)$ thus gives as a proportion of the total population the number of individuals below $\zeta$ in subgroups 1 to $i$, that is, in the $i$ most deprived, or neediest, subgroups. $\sum_{k=1}^{i} D^{1}(k ; \zeta)$ can then be termed the cumulative headcount index at $\zeta$ for the $i$ neediest subgroups. The first-order dominance condition (23) requires that this cumulative headcount be greater in $A$ than in $B$, whatever the number $i$ of groups we wish to include, and at all common poverty lines $0 \leq \zeta \leq z(i)$. Note, however, that it does not require that each subgroup $k$ have independently more poor in $A$ than in $B$, nor does it require that the population headcount (with each subgroup being assigned its own particular poverty line) be greater in $A$ than in $B$.

To see this more clearly, consider the case of poverty comparisons involving only two groups of individuals, $K=2$, with $z(1)$ being the poverty line of the neediest group and $z(2)$ the poverty line of the less needy individuals. Multidimensional dominance is checked first by comparing the headcount of the poor in group 1 for poverty lines $\zeta$ between 0 and $z(1)$, and then by comparing the combined poverty headcounts of the two groups at all common poverty lines between 0 and $z(2)$. This is illustrated in Figure 7. where $\zeta(1)$ and $\zeta(2)$ denote the poverty lines at which poverty in each of the two subgroups is assessed. For $\Pi^{1}(z(1), z(2))$ dominance, we need to compare the global poverty headcount at all of the combinations of poverty lines on the $\zeta(1)$ axis (up to 
$z(1)$, that is, up to point $\mathrm{G}$ ) and on the 45 degree line (until point $\mathrm{E})$. Comparing poverty for the combination of poverty lines on the $\zeta(1)$ axis amounts to checking the sign of $\Delta D^{1}(1 ; \zeta)$ for $\zeta \in[0, z(1)]$. Comparing poverty for the combination of poverty lines on the 45 degree line (until point $\mathrm{E}$ ) amounts to checking the sign of $\sum_{k=1}^{2} \Delta D^{1}(k ; \zeta)$ for $\zeta \in[0, z(2)]$.

If the dominance conditions in (23) are met, then we obtain a very robust ordering of multidimensional poverty. Indeed, we can then assert with confidence that all of the multidimensional poverty indices contained in $\Pi^{1}(\zeta(1), \ldots, \zeta(K))$ will show more poverty in $A$ than in $B$, and this, regardless of the selection of any particular combination of poverty lines, so long as they belong to the set defined by $\zeta(k) \in[0, z(k)], k=1, \ldots, K$.

\subsection{Discontinuous poverty indices}

The dominance condition becomes more stringent, however, if we include in the analysis the headcount and other indices that are discontinuous at the poverty line (in the manner, for instance, of Bourguignon and Fields (1997)) and replace assumption (22) by the following:

$$
\pi_{1}(x) \geq \pi_{2}(x) \geq \ldots \geq \pi_{K}(x) \geq 0, \forall x .
$$

A larger class $\tilde{\Pi}^{1}(z(1), \ldots, z(K))$ of additive poverty indices then includes all the $P$ indices defined in equation (17) that satisfy assumptions (20), (21) and (24). The "traditional" headcount index, by which total poverty is measured by assigning each subgroup its own poverty line, belongs to $\tilde{\Pi}^{1}$ but not to $\Pi^{1}$. We thus expect the dominance conditions for $\tilde{\Pi}^{1}$ to be correspondingly more demanding. The definition of $\tilde{\Pi}^{1}(z(1), \ldots, z(K))$ leads to the following equivalence:

\section{Theorem 6 (First-order poverty dominance without continuity)}

$$
\begin{aligned}
& \Delta P(z(1), \ldots, z(K))>0, \\
& \forall P(z(1), \ldots, z(K)) \in \tilde{\Pi}^{1}(z(1), \ldots, z(K)) \\
& \text { iff } \quad\left\{\begin{array}{l}
\sum_{k=1}^{i} \Delta D^{1}(k ; \zeta)>0, \forall \zeta \in[0, z(i)] \text { and } \forall i=1, \ldots, K \\
\text { and } \sum_{k=1}^{i} \Delta D^{1}(k ; z(k))>0, \forall i=1, \ldots, K .
\end{array}\right.
\end{aligned}
$$

Proof: See the appendix.

The first condition in (25) is identical to the one already discussed in (23). In addition, we must check that the cumulative headcounts are positive when each group $k$ has its specific poverty line set to $z(k)$. That is the second condition in (25). In the twogroup case of Figure 7, this adds to the previously-discussed test locations on more test at point $\mathrm{F}$ on the figure. The dominance conditions are thus more demanding than before. More importantly, however, note also that the combinations of poverty lines over which the $\tilde{\Pi}^{1}(z(1), \ldots, z(K))$ ranking is robust are far more restricted than for the previous result: in fact, dominance by (25) ensures robustness only at the exact combination 
of lines $\{z(1), \ldots, z(K)\}$. To extend the results to all of the poverty lines $\zeta(k)$ contained in $[0, z(k)]$ as in (23), we must also check the sign of the cumulative headcount when each subgroup is assigned its specific poverty line, instead of a common value $\zeta$. This new condition would need to be checked for all combinations of poverty lines (other than $\{z(1), \ldots, z(K)\})$ for which we would wish the poverty ordering $\tilde{\Pi}^{1}$ to be robust. For the 2-group case, this requires checking for dominance at all of the combinations of poverty lines defined by the shaded area of Figure 7 . This is clearly a much more stringent condition than that stated in Theorem 5 .

As in the continuous case of section 2, it is possible to extend the above reasoning to any arbitrary order of dominance. For a given order of dominance $s$, we would assume continuity at the poverty line up to the $(s-1)^{\text {th }}$ order. We would also require conditions on the $s^{\text {th }}$ order derivative $\pi_{k}^{(s)}(y ; z(k))$, and on the ranking of these derivatives across population subgroups. For second-order dominance, this would require that $\pi_{1}^{2}(y) \geq$ $\ldots \geq \pi_{K}^{2}(y) \geq 0, \forall y$. Indices in $\Pi^{2}$ would then be convex in $y$ and thus decreasing in mean-preserving equalizing transfers of living standards. They would thus obey the Pigou-Dalton principle of transfers within each group. The convexity of $\pi_{k}(y)$, and thus the importance of the Pigou-Dalton principle of transfers, would also be assumed to be decreasing in $k$ and hence increasing in the needs of the subgroups. At a given $y$, the greater the needs of a subgroup of individuals, the greater the beneficial poverty effect of a mean-preserving equalizing transfer within that subgroup.

The dominance conditions would then use $D^{2}(k ; \zeta)$ - which is the average poverty gap in subgroup $k$ for a poverty line $\zeta$, times the population share of subgroup $k \sum_{k=1}^{i} D^{2}(k ; \zeta)$ - and would cumulate it across the $i$ neediest subgroups. When this cumulative average poverty gap is greater in $A$ than in $B$, whatever the number $i$ of neediest subgroups included, and at all common poverty lines $0 \leq \zeta \leq z(i)$, poverty in $A$ is unambiguously greater than in $B$ for all of the indices in $\Pi^{2}(\zeta(1), \ldots, \zeta(K))$ and at all of the poverty lines $\zeta \in[0, z(i)]$. For the 2-group case, the graphical combinations of poverty lines over which this condition must be tested are the same as in the discussion of Figure 7 for condition $(23)^{17}$.

\subsection{Estimation}

As for section 3.1 in the case of continuous indicators of well-being, suppose that we have a random sample of $N$ independently and identically distributed observations drawn from the joint distribution of membership in group $k$ and indicator of well-being $x$. We can write these observations, drawn from a population $L$, as $\left(k_{i}^{L}, x_{i}^{L}\right), i=1, \ldots, N$. A natural estimator of the sum of the dominance curves $\sum_{k=1}^{j} D^{s}(k ; x)$ is then:

$$
\frac{1}{N} \sum_{i=1}^{N}\left(z_{k_{i}^{L}}-x_{i}^{L}\right)_{+}^{s} I\left(k_{i}^{L} \leq j\right) .
$$

\footnotetext{
${ }^{17}$ The details of this and extensions to higher-order dominance can be found in Duclos and Makdissi (2000).
} 
Expression (26) has again the convenient property of being a simple sum of IID variables. We can therefore use the result of Theorem 3 to show its asymptotic sampling distribution simply by replacing $\left(z_{y}\left(x^{L}\right)-y^{L}\right)_{+}^{\alpha_{y}}\left(z_{x}\left(x^{L}\right)-x^{L}\right)_{+}^{\alpha_{x}}$ by $\left(z_{k^{L}}-y^{L}\right)_{+}^{s} I\left(k^{L} \leq j\right)$ in (10), for $L=A, B$. An exactly analogous exercise can be done for the sampling distribution of critical poverty frontiers indexed over the subgroups $k$.

\subsection{Examples}

Our first example for this section handles the equivalence scale problem with the method first suggested by Atkinson (1991). We ask the question, "which type of transfer payment reduces poverty more in Romania, child allowances or social security pensions?" Because the answer can easily be influenced by the choice of equivalence scale, we will avoid that choice altogether. Instead, we use bivariate dominance tests where the second variable is household size, an indicator of greater needs. The neediest group is households with six or more people, $\frac{18}{1}$ the next neediest contains households with five people, etc.. The data come from the Romania Integrated Household Survey (Government of Romania, 1994). The other well-being variable is household income, plus the relevant transfer payment (child allowances or social security pensions). We have standardized these payments so that they have the same mean, thus ensuring that the tests do not merely reflect that fact that one program is very large relative to the other.

Table 4 gives the $t$-statistics for the differences in the dominance curves of the neediest group, the two neediest groups, etc., up to the entire sample, as required by theorem 5. The difference is the dominance curve for income plus child allowances minus that for income plus social security pensions. For large households, child allowances clearly reduce poverty by more than social security payments, but this result is reversed abruptly once we include households with two people, where the dominance curves now cross, and where social security payments appear to be more beneficial to poorer households. The same pattern holds for $s=2$ and $s=3$ (involving the "poverty gap" and "poverty severity" curves, respectively), suggesting that we cannot make any robust statement as to the poverty reducing impact of these two transfer payments without excluding households of 2 and 1 persons.

Our second example in this section considers a case in which poverty is measured on two dimensions, household expenditures per capita and literacy, the latter of which is discrete. We consider the change in poverty in Peru between 1985 and 1994, as measured by the Encuesta Nacional de Níveles de Vida in those two years. Table 5 is similar to Table 4, but the groups are now defined by literacy. We assume that, for a given level of expenditure, those who cannot read and write have lower well-being than those who can. Thus, the first group is the illiterate population. The $t$-statistics are for the 1985 dominance curve minus the 1994 curve. Except for the first ordinate for group 1, which just misses the five percent significance level (and for which there are very few observations

\footnotetext{
${ }^{18}$ There are very few households with more than six people in the sample.
} 
in the sample), there is a clear worsening of poverty as measured by expenditures and literacy.

\section{Conclusion}

In this paper, we have shown that it is possible to make sensible comparisons of poverty when poverty is measured in multiple dimensions. These comparisons have several attractive features:

1. In the spirit of the stochastic dominance literature, they can be tested for robustness over broad classes of poverty indices. A special consideration for the multivariate case is whether poverty is defined as the intersection or union of poverty in each dimension. The methods that we describe are valid for both, as well as for intermediate cases in which the poverty line in one dimension is a non-increasing function of well-being in other dimensions.

2. The poverty comparisons can be tested for robustness over a broad choice of frontiers of poverty lines in each dimension. Alternatively, one can estimate a critical poverty frontier up to which multidimensional poverty dominance necessarily holds.

3. Poverty comparisons are possible for a mixture of discrete and/or continuous welfare variables.

4. The comparisons involve statistical tests, that make use of the sampling distribution of multidimensional poverty estimators.

The importance of these methods rests on two considerations. The first is ethical and rests on the widespread acknowledgement that well-being and poverty are multidimensional in nature. We take this as given, based either on Sen's philosophical arguments that poverty should be viewed in terms of capabilities and functionings, or on the more narrow recognition that a person's well-being has dimensions that cannot be purchased and that transcend levels of income. The second consideration is practical: to what extent will multidimensional comparisons differ from unidimensional ones? Given the relatively weak correlations that are often observed between income and other welfare variables, it should not be surprising to find cases where poverty comparisons in multiple dimensions differ from comparisons in only one of those dimensions, something that we found repeatedly in preparing the examples for this paper.

More generally, we have shown that our multidimensional comparisons can also differ from univariate comparisons in each dimension in two ways. One could find dominance in each dimension separately if the margins of the dominance surfaces differ in each dimension, but may not find multidimensional dominance if the surfaces cross in the surfaces' interiors. This draws attention to the importance of capturing the incidence of multiple deprivation. Alternatively, the one-dimensional dominance curves may cross, 
ruling out univariate dominance, but the interiors of the multidimensional surfaces may be uniformly different, allowing multivariate dominance for intersection or intermediate poverty definitions. How important empirically these two possibilities are remains to be firmly established. Our admittedly limited experience based on comparisons of the DHS surveys is that the first is rare, while the second is fairly common. But a firm sense of the importance of our more general methods must await further practice with other samples and other variables.

\section{Appendix}

\section{Proof of Theorem 1 .}

We proceed by first integrating equation (1) by parts with respect to $x$. This gives:

$$
\begin{aligned}
P\left(z_{x}(y), z_{y}\right) & =\left.\int_{0}^{z_{y}}\left[\pi\left(x, y ; z_{x}(y), z_{y}(x)\right) F(x \mid y)\right]\right|_{0} ^{z_{x}(y)} f(y) d y \\
& -\int_{0}^{z_{y}} \int_{0}^{z_{x}(y)} \pi^{x}\left(x, y ; z_{x}(y), z_{y}(x)\right) F(x \mid y) f(y) d x d y .
\end{aligned}
$$

The first term on the right-hand-side of (27) is zero since $F(x=0 \mid y)=0$ and since we assumed that $\pi\left(z_{x}(y), y ; z_{x}(y), y\right)=0$. (Hence, it is here that the continuity assumption at the set of poverty lines is technically useful.) To integrate by parts with respect to $y$ the second term, define a general function $K(y)=\int_{0}^{g(y)} h(x, y) l(x, y) d x$ and note that:

$$
\begin{aligned}
\frac{d K(y)}{d y} & =g^{(1)}(y) h(g(y), y) l(g(y), y) \\
& +\int_{0}^{g(y)} \frac{\partial h(x, y)}{\partial y} l(x, y) d x \\
& +\int_{0}^{g(y)} h(x, y) \frac{\partial l(x, y)}{\partial y} d x
\end{aligned}
$$

Reordering (28) and integrating it from 0 to $c$, we find:

$$
\begin{aligned}
& -\int_{0}^{c} \int_{0}^{g(y)} h(x, y) \frac{\partial l(x, y)}{\partial y} d x d y \\
& =-K(c)+K(0)+\int_{0}^{c} g^{(1)}(y) h(g(y), y) l(g(y), y) d y \\
& +\int_{0}^{c} \int_{0}^{g(y)} \frac{\partial h(x, y)}{\partial y} l(x, y) d x d y .
\end{aligned}
$$

Now replace in (29) $c$ by $z_{y}, g(y)$ by $z_{x}(y), h(x, y)$ by $\pi^{x}\left(x, y ; z_{x}(y), z_{y}(x)\right), l(x, y)$ by $F(x, y)$ and $K(y)$ by its definition $K(y)=\int_{0}^{g(y)} h(x, y) l(x, y) d x$. This gives: 


$$
\begin{aligned}
P\left(z_{x}(y), z_{y}\right) & =-\int_{0}^{z_{x}\left(z_{y}\right)} \pi^{x}\left(x, z_{y} ; z_{x}\left(z_{y}\right), z_{y}(x)\right) D^{1,1}\left(x, z_{y}\right) d x \\
& +\int_{0}^{z_{y}} z_{x}^{(1)}(y) \pi^{x}\left(z_{x}(y), y ; z_{x}(y), y\right) D^{1,1}\left(z_{x}(y), y\right) d y \\
& +\int_{0}^{z_{y}} \int_{0}^{z_{x}(y)} \pi^{x y}\left(x, y ; z_{x}(y), z_{y}(x)\right) D^{1,1}(x, y) d x d y
\end{aligned}
$$

For the sufficiency of condition (6), recall that $z_{x}^{(1)}(y) \leq 0, \pi^{x} \leq 0$, and $\pi^{x y} \geq 0$, with strict inequalities for either of these inequalities over at least some inner ranges of $x$ and $y$. Hence, if $\Delta D^{1,1}(x, y)>0$, for all $y \in\left[0, z_{y}\right]$ and for all $x \in\left[0, z_{x}(y)\right]$, then it must be that $\Delta P\left(\zeta_{x}(y), \zeta_{y}\right)>0$ for all of the indices and of the sets of poverty lines specified in Theorem 1 .

For the necessity part, assume that $\Delta D^{1,1}(x, y) \leq 0$ for an area defined over $x \in$ $\left[c_{x}^{-}, c_{x}^{+}\right]$and $y \in\left[c_{y}^{-}, c_{y}^{+}\right]$, with $c_{x}^{+} \leq z_{y}$ and $c_{x}^{+} \leq z_{x}(y)$. Then any of the poverty indices in $\ddot{\Pi}^{1,1}$ for which $\pi^{x y}<0$ over that area and for which $\pi^{x}\left(x, z_{y} ; z_{x}\left(z_{y}\right), z_{y}(x)\right)=$ $\pi^{x}\left(z_{x}(y), y ; z_{x}(y), y\right)=0$ will indicate either that $\Delta P=0$ or that $\Delta P<0$. Condition (6) is thus also a necessary condition for the ordering specified in Theorem 1 .

\section{Proof of Theorem 2.}

Integrating (30) once more by parts with respect to $x$, and imposing the continuity conditions characterizing the indices $\ddot{\Pi}^{2,1}\left(z_{x}(y), z_{y}\right)$ in (7), we find:

$$
\begin{aligned}
P\left(z_{x}(y), z_{y}\right) & =\int_{0}^{z_{x}\left(z_{y}\right)} \pi^{x x}\left(x, z_{y}\right) D^{2,1}\left(x, z_{y}\right) d x \\
& +\int_{0}^{z_{y}} \pi^{x y}\left(z_{x}(y), y\right) D^{2,1}\left(z_{x}(y), y\right) d y \\
& -\int_{0}^{z_{y}} \int_{0}^{z_{x}(y)} \pi^{x x y}(x, y) D^{2,1}(x, y) d x d y
\end{aligned}
$$

The rest of the proof is as for Theorem 1 .

\section{Proof of Theorem 3 .}

A natural estimator of the multidimensional FGT indices $P\left(z_{x}(y), z_{y} ; \alpha_{x}, \alpha_{y}\right)$ is given by:

$$
\begin{aligned}
& \hat{P}_{L}\left(z_{x}(y), z_{y} ; \alpha_{x}, \alpha_{y}\right) \\
& \quad=\int_{0}^{z_{y}} \int_{0}^{z_{x}(y)}\left(z_{y}(x)-y\right)^{\alpha_{y}}\left(z_{x}(y)-x\right)^{\alpha_{x}} d \hat{F}_{L}(x, y) \\
& \quad=\frac{1}{N} \sum_{i=1}^{N}\left(z_{y}\left(x_{i}^{L}\right)-y_{i}^{L}\right)^{\alpha_{y}}\left(z_{x}\left(y_{i}^{L}\right)-x_{i}^{L}\right)^{\alpha_{x}} I\left(y_{i}^{L} \leq z_{y}\left(x_{i}^{L}\right)\right) I\left(x_{i}^{L} \leq z_{x}\left(y_{i}^{L}\right)\right) \\
& \quad=\frac{1}{N} \sum_{i=1}^{N}\left(z_{y}\left(x_{i}^{L}\right)-y_{i}^{L}\right)_{+}^{\alpha_{y}}\left(z_{x}\left(y_{i}^{L}\right)-x_{i}^{L}\right)_{+}^{\alpha_{x}}
\end{aligned}
$$


A special case of (32) is the estimator of the dominance surface in (9), obtained simply by specifying $z_{x}(y)=z_{x}, z_{y}(x)=z_{y}$, and $\alpha_{x}=s_{x}-1$ and $\alpha_{y}=s_{y}-1$.

For each distribution, the existence of the appropriate population moments of order 1 lets us apply the law of large numbers to (32), thus showing that $\hat{P}_{K}\left(z_{x}(y), z_{y} ; \alpha_{x}, \alpha_{y}\right)$ is a consistent estimator of $\left.P_{K}\left(z_{x}(y), z_{y} ; \alpha_{x}, \alpha_{y}\right)\right)$. Given also the existence of the population moments of order 2 , the central limit theorem shows that the estimator in (32) is root- $N$ consistent and asymptotically normal with asymptotic covariance matrix given by (10). When the samples are dependent, the covariance between the estimator for $A$ and for $B$ is also provided by (10).

Theorem 3 thus provides the formula needed to estimate the sampling variability of any point on the dominance surfaces and for any choice of multidimensional poverty lines in the multidimensional FGT poverty indices. Extension of the result of Theorem 3 to any additive multidimensional poverty indices is straightforward, and simply requires substituting in (10) the relevant functions $\pi\left(x, y ; z_{x}(y), z_{y}(x)\right)$ for $\left(\left(z_{y}(x)-y\right)_{+}^{\alpha_{y}}\left(z_{x}(y)-\right.\right.$ $x)_{+}^{\alpha_{x}}$.

\section{Proof of Theorem 4 ,}

The proof can be established along the lines of the proof of Theorem 3 in Davidson and Duclos (2000). To see this, note that the conditions of Theorem 4 assume that the appropriate joint population moments exist, and that the critical frontier $\zeta_{x}^{+}(y)$ also exists in the population. Furthermore, since this frontier is assumed to be where the dominance surfaces exactly cross, we have that $\Delta P^{x}\left(\zeta_{x}^{+}(y), y ; s_{x}-1, s_{y}-1\right)<0$. Note that this derivative is given by $\delta \cdot\left(P_{A}\left(\zeta_{x}^{+}(y), y ; s_{x}-2, s_{y}-1\right)-P_{B}\left(\zeta_{x}^{+}(y), y ; s_{x}-2, s_{y}-1\right)\right)$, with $\delta=s_{x}-1$ when $s_{x}>1$, and $\delta=1$ when $s_{x}=1$. When $s_{x}=1$, we also have $P_{L}\left(\zeta_{x}^{+}(y), y ;-1, s_{y}-1\right)=E\left[\left(y-y^{L}\right)_{+}^{\left(s_{y}-1\right)} \mid x=\zeta_{x}^{+}(y)\right] f_{x}\left(\zeta_{x}^{+}(y)\right), L=A, B$.

Again, the elements of the asymptotic covariance matrix can be estimated consistently by simply using their sample estimates. Estimating $P\left(\zeta_{x}^{+}(y), y ; s_{x}-2, s_{y}-1\right)$ is also easily done when $s_{x}>1$. Estimating $E\left[\left(y-y^{L}\right)_{+}^{\left(s_{y}-1\right)} \mid x=\zeta_{x}^{+}(y)\right] f_{x}\left(\zeta_{x}^{+}(y)\right)$ is slightly more complicated, but can be done consistently using non-parametric regression procedures. In particular, we use in the illustration a Gaussian kernel, $K(u)=$ $(2 \pi)^{-0.5} \exp ^{-0.5 u^{2}}$, and estimate $E\left[\left(y-y^{L}\right)_{+}^{\left(s_{y}-1\right)} \mid x=\zeta_{x}^{+}(y)\right] f_{x}\left(\zeta_{x}^{+}(y)\right)$ as:

$$
(n h)^{-1} \sum_{i=1}^{n} K\left(\frac{\zeta_{x}^{+}(y)-x_{i}^{L}}{h}\right)\left(y-y_{i}^{L}\right)_{+}^{\left(s_{y}-1\right)} .
$$

\section{Proof of Theorem 5 .}

We first use (17) to integrate by parts the difference $\Delta P$. We find:

$$
\begin{aligned}
\Delta P & =\sum_{k=1}^{K} \pi_{k}(z(k)) \Delta D^{1}(k ; z(k)) \\
& -\sum_{k=1}^{K} \int_{0}^{z(k)} \pi_{k}^{(1)}(x) \Delta D^{1}(k ; x) d x .
\end{aligned}
$$


Recall the continuity assumption that $\pi_{k}(z(k))=0, \forall k$. For $\Delta P>0$, we thus need to show that

$$
\sum_{k=1}^{K} \int_{0}^{z(k)} \pi_{k}^{(1)}(x) \Delta D^{1}(k ; x) d x<0 .
$$

Recall that $\pi_{k}^{(1)}(x)=0$ if $x>z(k)$; combined with (20), we can then rewrite (35) as:

$$
\int_{0}^{z(1)} \sum_{k=1}^{K} \pi_{k}^{(1)}(x) \Delta D^{1}(k ; x) d x<0 .
$$

The inner sum in (36) can be rewritten as:

$$
\begin{aligned}
\sum_{k=1}^{K} \pi_{k}^{(1)}(x) \Delta D^{1}(k ; x) & = \\
\pi_{K}^{(1)}(x) \sum_{l=1}^{K} \Delta D^{1}(l ; x) & +\left(\pi_{K-1}^{(1)}(x)-\pi_{K}^{(1)}(x)\right) \sum_{l=1}^{K-1} \Delta D^{1}(l ; x) \\
+\ldots & +\left(\pi_{1}^{(1)}(x)-\pi_{2}^{(1)}(x)\right) \Delta D^{1}(1 ; x)
\end{aligned}
$$

Denoting $\pi_{K+1}^{(1)}(x) \equiv 0$, we can thus rewrite the right-hand-side of (36) as

$$
\int_{0}^{z(1)} \sum_{i=1}^{K}\left[\left(\pi_{i}^{(1)}(x)-\pi_{i+1}^{(1)}(x)\right) \sum_{k=1}^{i} \Delta D^{1}(k ; x)\right] d x .
$$

Note that by the definition of the class of indices $\Pi^{1}(\zeta(1), \ldots, \zeta(K)), \pi_{i}^{(1)}(x)-$ $\pi_{i+1}^{(1)}(x) \leq 0, \forall i=1, \ldots, K$, with strict inequality for some values of $i$ over some range of $x \in[0, \zeta(i)]$ (for the indices to be non-degenerate). Hence, if $\sum_{k=1}^{i} \Delta D^{1}(k ; \zeta)>$ $0, \forall \zeta \in[0, z(i)]$ and $\forall i=1, \ldots, K$, then it must be that (35) holds for all $P(z(1), \ldots, z(K))$ $\in \Pi^{1}(z(1), \ldots, z(K))$. But this also implies that $\Delta P(\zeta(1), \ldots, \zeta(K))>0, \forall P(\zeta(1), \ldots, \zeta(K))$ $\in \Pi^{1}(\zeta(1), \ldots, \zeta(K))$, and $\forall \zeta(k) \in[0, z(k)], k=1, \ldots, K$. This proves the sufficiency of condition (23).

For the necessity part, it suffices to consider any particular case in which $\sum_{k=1}^{i} \Delta D^{1}(k ; \zeta) \leq$ 0 , for some $\zeta \in\left[z^{-}(i), z^{+}(i)\right]$ and for some value of $i$. Consider then a poverty index that belongs to $\Pi^{1}(z(1), \ldots, z(K))$ such that $\pi_{k}^{(1)}(x)-\pi_{k+1}^{(1)}(x)=0$ everywhere, except for $k=i$ and over that range $\zeta \in\left[z^{-}(i), z^{+}(i)\right]$ over which $\sum_{k=1}^{i} \Delta D^{1}(k ; \zeta) \leq 0$. Then, by (40), $\Delta P \leq 0$ for that index, which therefore shows the necessity of condition (23).

Proof of Theorem 6 .

Consider again equation (34):

$$
\begin{aligned}
\Delta P & =\sum_{k=1}^{K} \pi_{k}(z(k)) \Delta D^{1}(k ; z(k)) \\
& -\sum_{k=1}^{K} \int_{0}^{z(k)} \pi_{k}^{(1)}(x) \Delta D^{1}(k ; x) d x .
\end{aligned}
$$


The second part of condition (15) guarantees the non-negativity of the second part of (41), as shown before in the proof of Theorem 5. Denoting again $\pi_{K+1}^{(1)}(y) \equiv 0$, rewrite the first part of (41) as:

$$
\sum_{i=1}^{K}\left[\left(\pi_{i}(z(i))-\pi_{i+1}(z(i+1))\right) \sum_{k=1}^{i} \Delta D^{1}(k ; z(i))\right] .
$$

Note that by the definition of the class of indices $\tilde{\Pi}^{1}(z(1), \ldots, z(K)), \pi_{i}(z(i))-$ $\pi_{i+1}(z(i+1)) \leq 0, \forall i=1, \ldots, K$. Hence, if $\sum_{k=1}^{i} \Delta D^{1}(k ; z(k))>0, \forall i=1, \ldots, K$, then the first part on the right-hand-side of (41) is also non-negative. The combination of the first and of the second parts of condition (25) guarantees that $\Delta P>0$.

The necessity of condition (15) proceeds as for the proof of Theorem 5 .

\section{References}

[1] Atkinson, A.B. (1987). "On the Measurement of Poverty”, Econometrica, 55, 749764.

[2] Atkinson, A.B. (1991). "Measuring Poverty and Differences in Family Composition”, Economica, 59, 1-16.

[3] Atkinson, A.B. and F. Bourguignon (1982). "The Comparison of MultiDimensional Distributions of Economic Status", chapter 2 in Social Justice and Public Policy, Harvester Wheatsheaf, London.

[4] Atkinson, A.B. and F. Bourguignon (1987). "Income Distribution and Differences in Needs", in G.R. Feiwel, ed., Arrow and the foundations of the theory of economic policy, New York Press, New York, 350-70.

[5] Bourguignon, F., and S. R. Chakravarty (1998). "The measurement of multidimensional poverty", Delta working paper, 98-12.

[6] Bourguignon, F. and G. Fields (1997). "Discontinuous Losses from Poverty, Generalized P Measures, and Optimal Transfers to the Poor", Journal of Public Economics, 63,155-175.

[7] Chakravarty, S.R. (1983). "A New Index of Poverty", Mathematical Social Sciences, 6, 307-313.

[8] Chambaz, C. and E. Maurin (1998), "Atkinson and Bourguignon Dominance Criteria: Extended and Applied to the Measurement of Poverty in France", Review of Income and Wealth, 44, 77-124.

[9] Davidson, R. and J.-Y. Duclos (2000). "Statistical Inference for Stochastic Dominance and the for the Measurement of Poverty and Inequality", Econometrica, 68, $1435-1465$. 
[10] Duclos, J.-Y. and P. Makdissi (2000). "Sequential Stochastic Dominance and the Robustness of Poverty Orderings", Cahier de recherche, Département d'économique, Université Laval.

[11] Fishburn, P.C. and R.D. Willig (1984). "Transfer Principles in Income Redistribution”, Journal of Public Economics, 25, 323-328.

[12] Foster, J.E., J. Greer and E. Thorbecke (1984). "A Class of Decomposable Poverty Measures", Econometrica, 52 (3), 761-776.

[13] Foster, J.E. and A.F. Shorrocks (1988a). "Poverty Orderings", Econometrica, 56, $173-177$.

[14] Foster, J.E. and A.F. Shorrocks (1988b). "Poverty Orderings and Welfare Dominance", Social Choice Welfare, 5, 179-198.

[15] Foster, J.E. and A.F. Shorrocks (1988c). "Inequality and Poverty Orderings", European Economic Review, 32, 654-662.

[16] Government of Romania (1994). Romania Integrated Household Survey.

[17] Grosh, B. and P. Glewwe (1998). "Data Watch: The World Bank's Living Standards Measurement Study Household Surveys”, Journal of Economic Perspectives, 12, 187-96.

[18] Howes, S. (1996). "A new test for inferring dominance from sample data", draft.

[19] Jenkins, S.P. and P.J. Lambert (1993). "Ranking Income Distributions when Needs Differ", Review of Income and Wealth, 39, 337-356.

[20] Sahn, David E. and David C. Stifel (2000). "Poverty Comparisons Over Time and Across Countries in Africa," World Development, Vol. 28, Number 12.

[21] Sahn, David E., David Stifel, and Stephen Younger (1999). "Inter-temporal Changes in Welfare: Preliminary Results from Nine African Countries," CFNPP Working Paper \#94.

[22] Sen, A.K. (1985), Commodities and Capabilities, North-Holland, Amsterdam.

[23] Shorrocks, A.F., and J. Foster (1987). "Transfer Sensitive Inequality Measures", Review of Economic Studies, LIV, 485-497.

[24] United Nations Development Program (1990). Human Development Report. New York: Oxford University Press.

[25] Watts, H.W. (1968), "An Economic Definition of Poverty", in D.P. Moynihan (ed.), On Understanding Poverty, New York: Basic Books. 
Figure 1: Union and intersection poverty indices

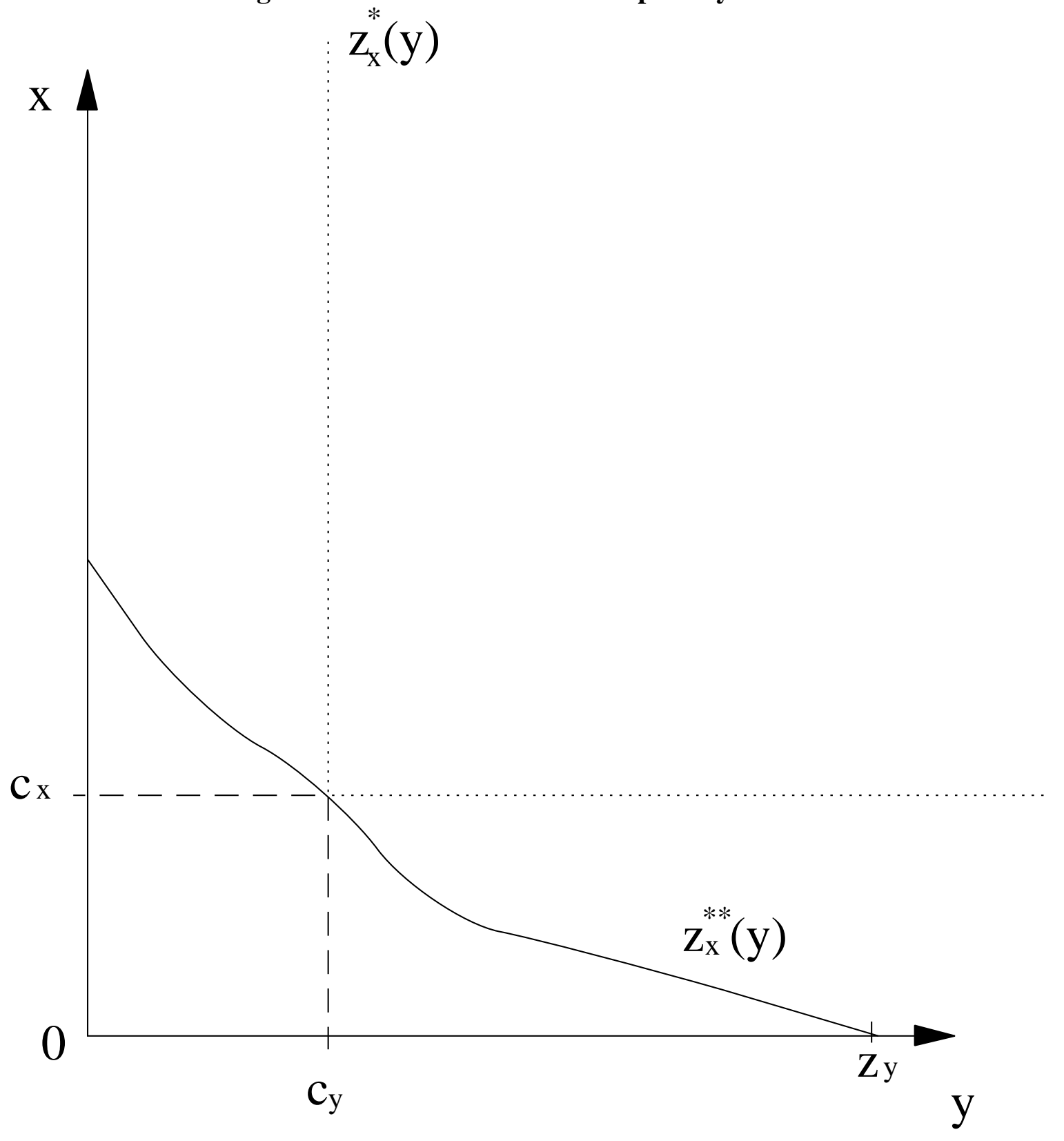


Figure 2: Dominance surface for Ghanaian children, 1989

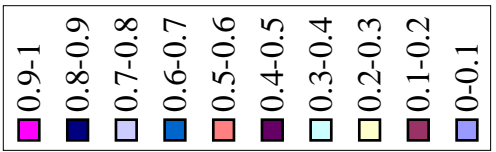

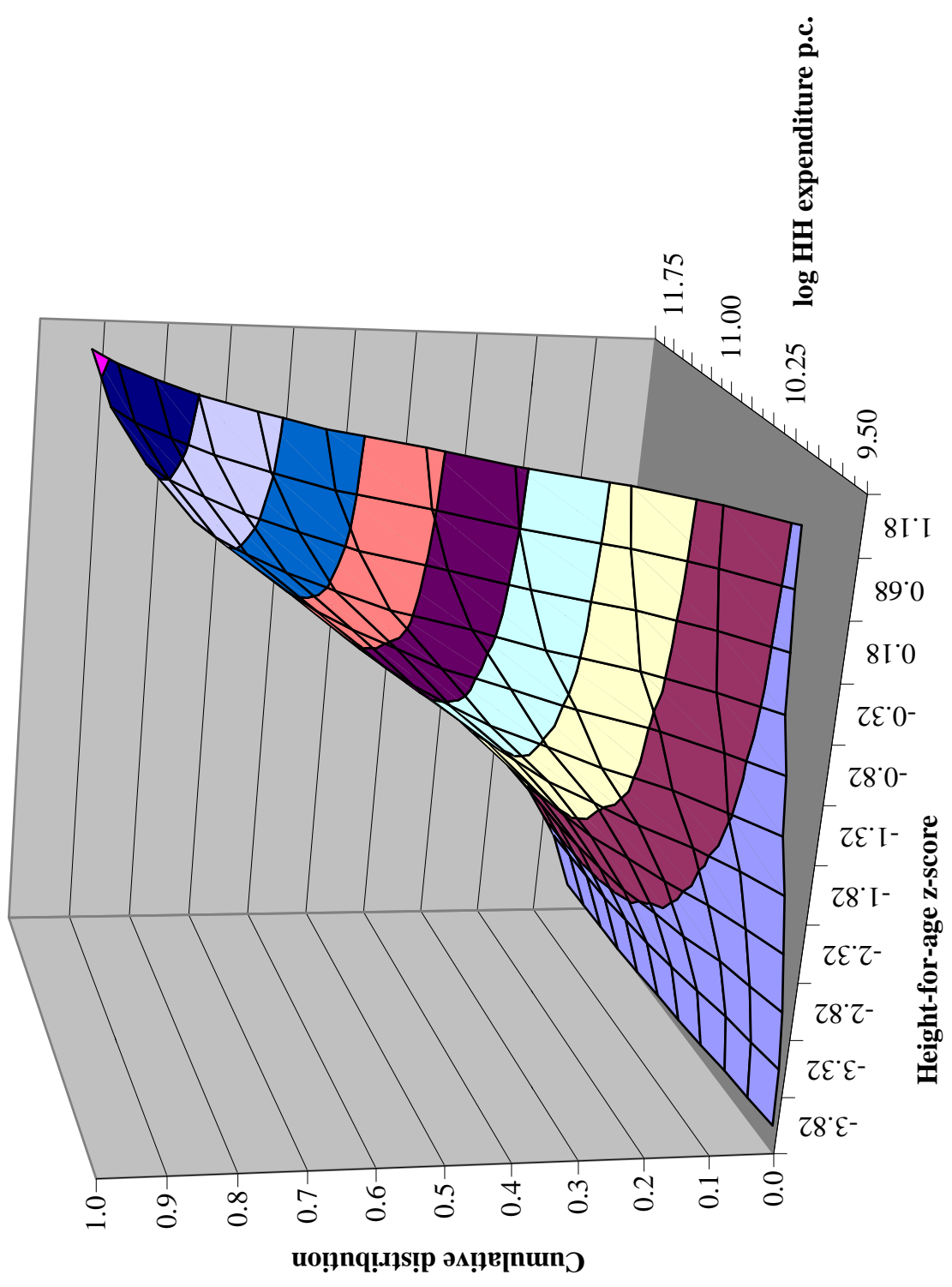


Figure 3: Example of difference in dominance surfaces, intersection dominance without marginal dominance

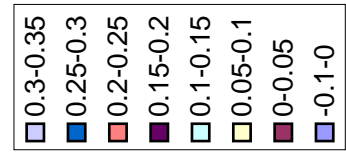

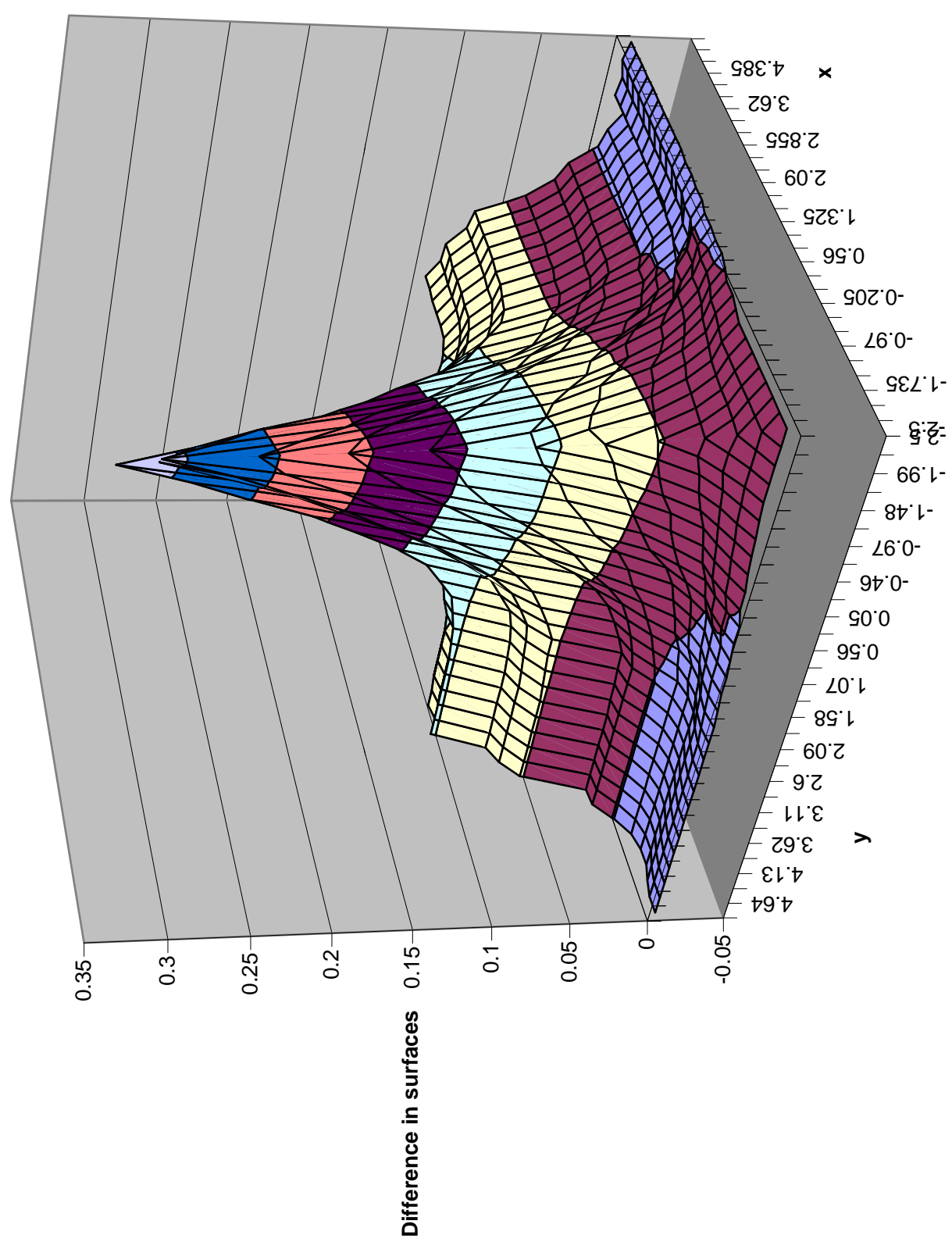


Figure 4: Urban minus Rural Dominance Surface for Viet Nam

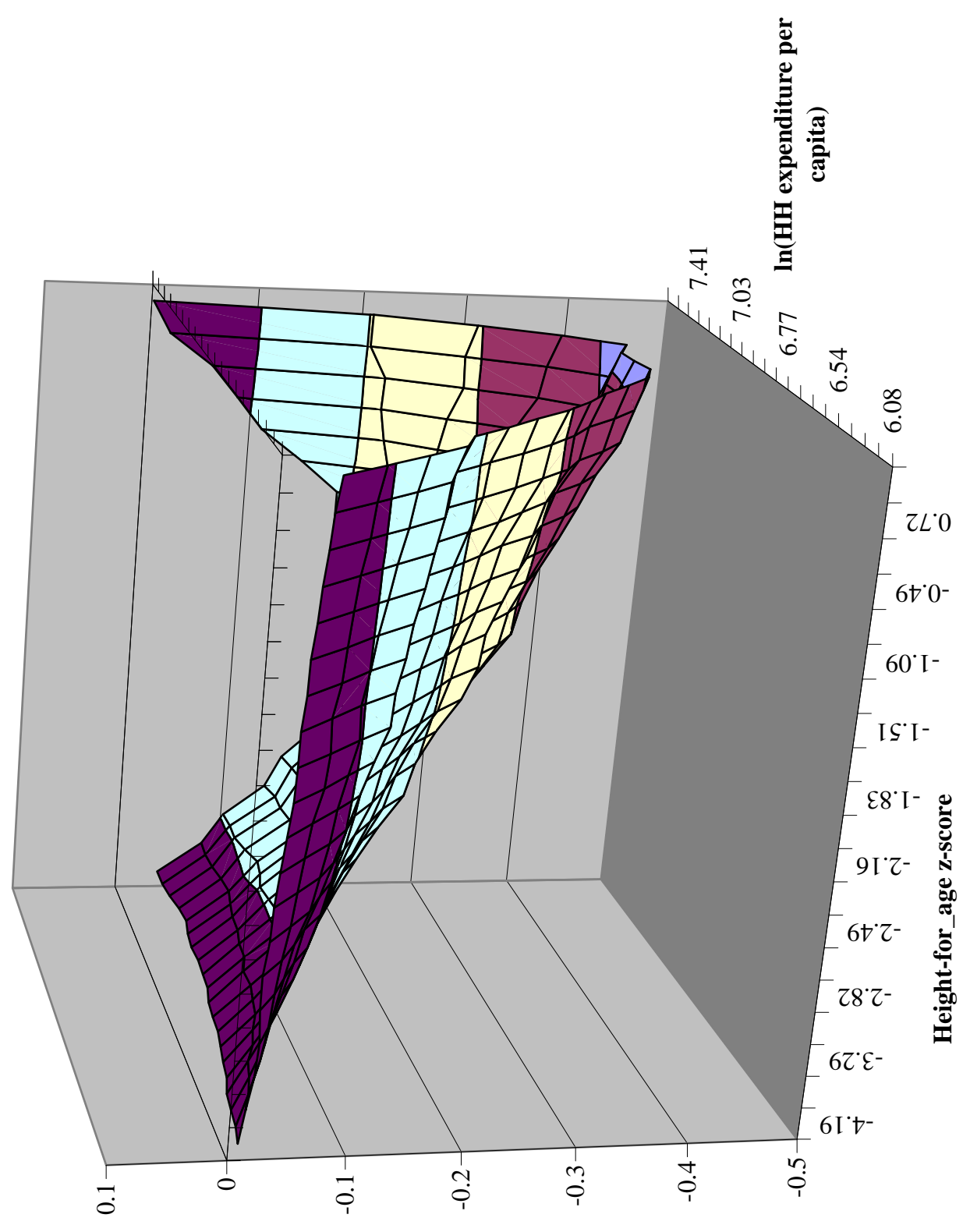


Figure 5: Test results for difference between 1993 and 1998 first-order dominance surfaces for Ghanaian children

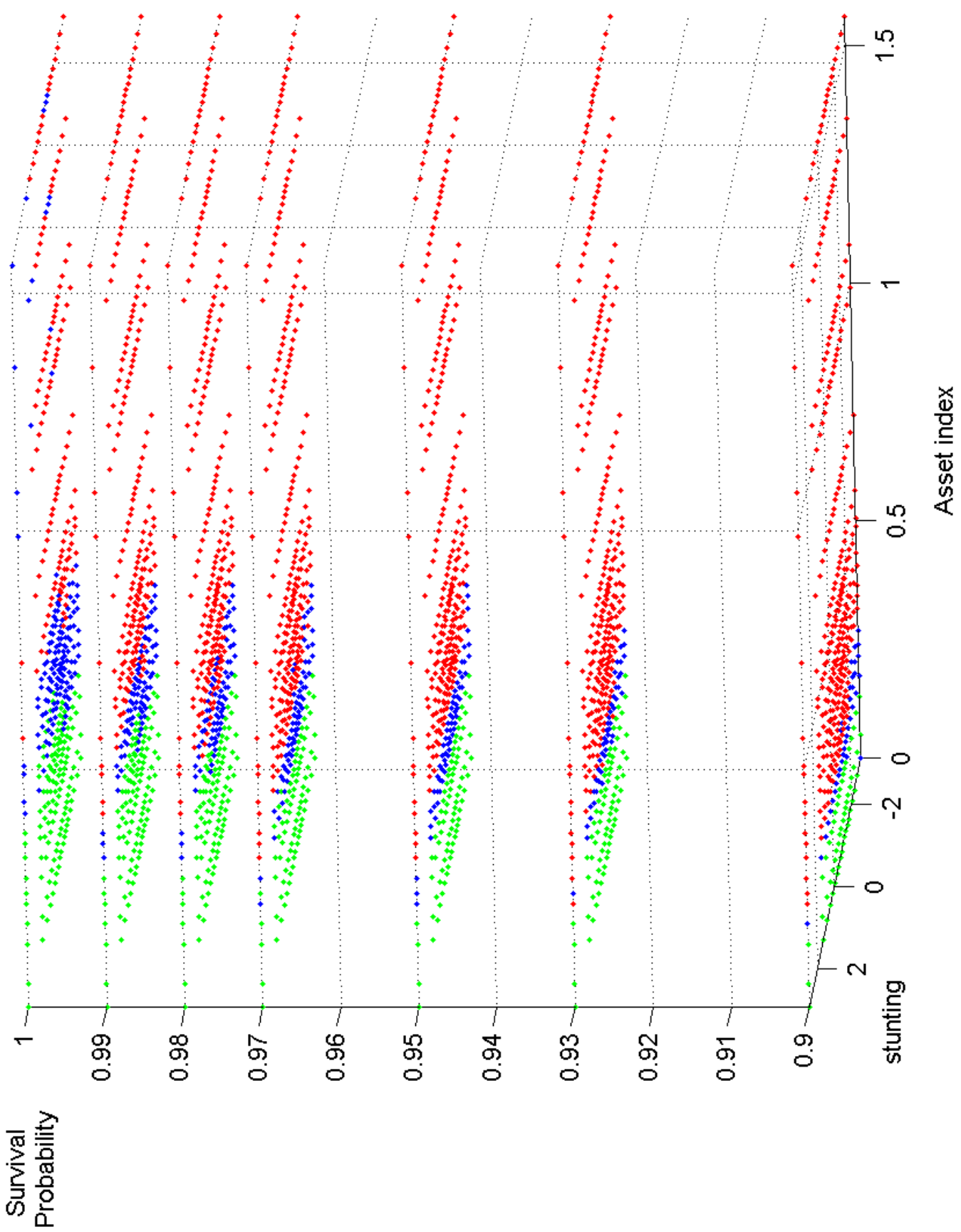


Figure 6: Critical Poverty Frontier, Children's Wasting and Survival probability, Madagascar and Egypt

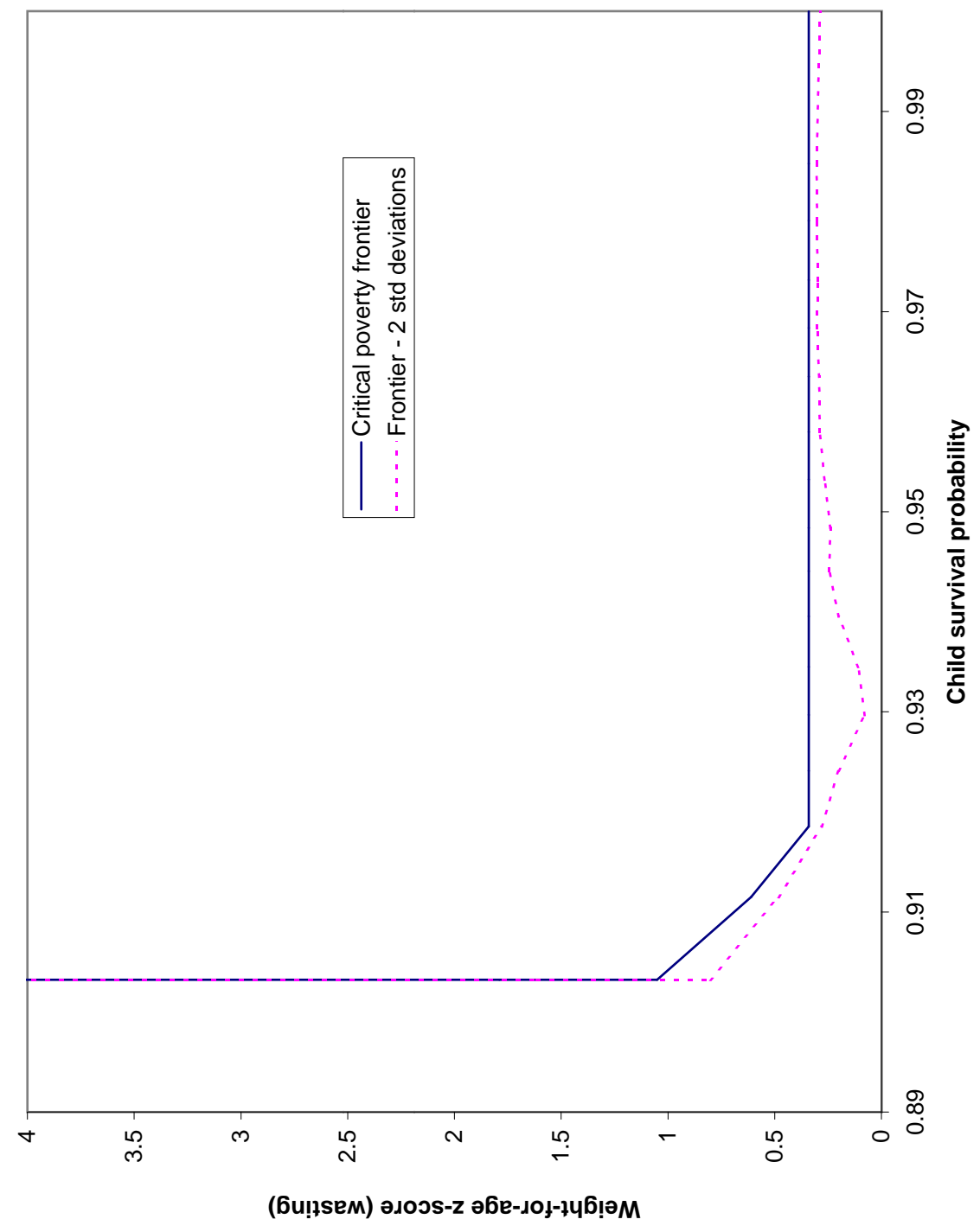


Figure 7: Domain for dominance testing

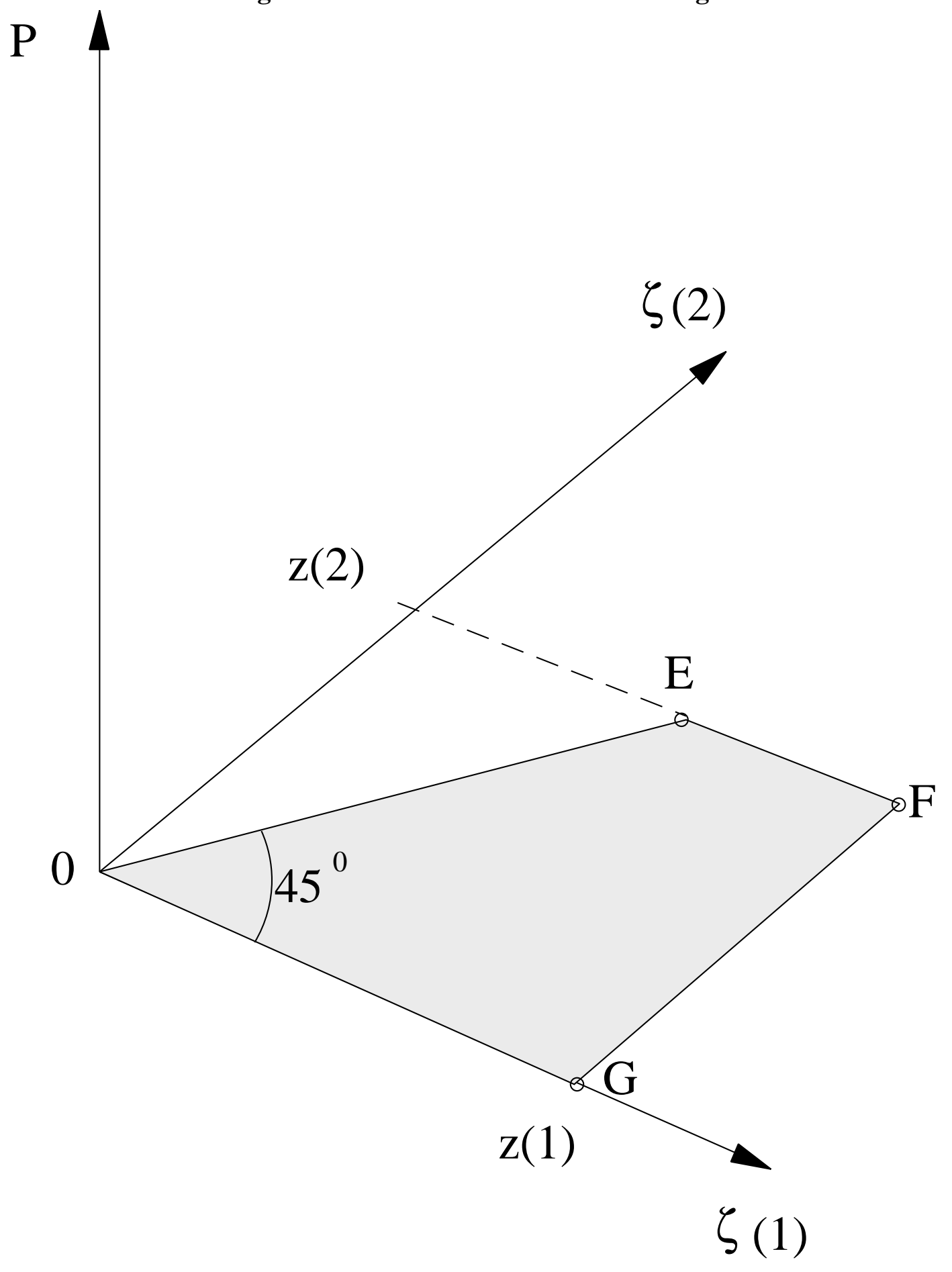


Table 1: Test results for difference between dominance surfaces for urban and rural children in Viet Nam, 1993

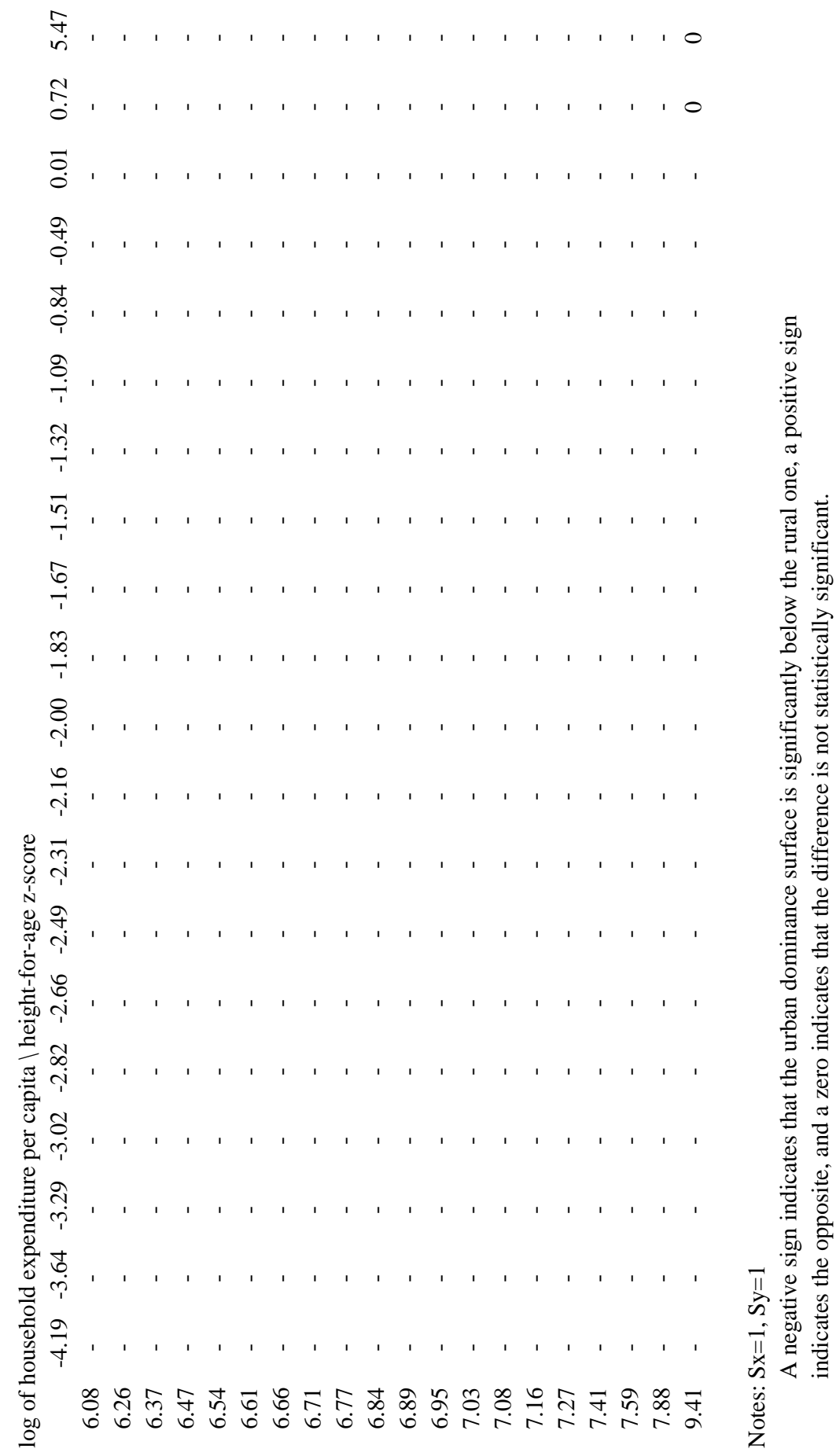




\section{Table 2: Test results for difference between dominance surfaces for chil-}

dren in Cameroon and Madagascar, 1997

\begin{tabular}{ccccccccccc}
\multicolumn{1}{c}{ Height-for-age z-score $\backslash$ Survival probability } \\
\multicolumn{1}{c}{0.83} & 0.86 & 0.88 & 0.89 & 0.90 & 0.91 & & 0.99 & 1.00 \\
-4.19 & - & - & 0 & 0 & - & - & $\ldots$ & - & - \\
-3.66 & 0 & 0 & 0 & 0 & - & - & $\ldots$ & - & - \\
-3.35 & 0 & - & - & - & - & - & $\ldots$ & - & - \\
-3.13 & 0 & - & - & - & - & - & $\ldots$ & - & - \\
-2.88 & 0 & - & - & - & - & - & $\ldots$ & - & - \\
-2.66 & - & - & - & - & - & - & $\ldots$ & - & - \\
-2.50 & - & - & - & - & - & - & $\ldots$ & - & - \\
& $\ldots$ & $\ldots$ & $\ldots$ & $\ldots$ & $\ldots$ & $\ldots$ & $\ldots$ & $\ldots$ & $\ldots$ \\
0.46 & - & - & - & - & - & - & $\ldots$ & - & - \\
5.39 & - & - & - & - & - & - & $\ldots$ & - &.
\end{tabular}

Notes: $1 / \mathrm{Sx}=1, \mathrm{Sy}=1$

2/ A negative sign indicates that Madagascar's dominance surface is significantly above Cameroon's, a positive sign indicates the opposite, and a zero indicates that the difference is not statistically significant. 3/ The ellipses indicate that all intervening signs are negative. 


\section{Table 3: Test results for difference between dominance surfaces for chil- dren in Colombia and the Dominican Republic, 1995 and 1996}

\begin{tabular}{|c|c|c|c|c|c|c|c|c|c|c|c|c|}
\hline \multicolumn{13}{|c|}{ Height-for-age z-score \Survival probability } \\
\hline & 0.906 & 0.927 & 0.938 & 0.947 & 0.953 & & 0.985 & 0.987 & 0.989 & 0.991 & 0.995 & 1.000 \\
\hline-2.85 & - & - & - & - & - & $\ldots$ & - & - & - & 0 & 0 & 0 \\
\hline-2.36 & - & - & - & - & - & $\ldots$ & - & - & - & - & 0 & 0 \\
\hline-2.07 & - & - & - & - & - & $\ldots$ & - & - & - & - & 0 & 0 \\
\hline-1.85 & - & - & - & - & - & $\ldots$ & - & - & - & 0 & 0 & 0 \\
\hline-1.67 & - & - & - & - & - & $\ldots$ & - & - & - & 0 & 0 & 0 \\
\hline-1.47 & - & - & - & - & - & $\ldots$ & - & - & - & 0 & 0 & + \\
\hline-1.33 & - & - & - & - & - & $\ldots$ & - & - & - & 0 & 0 & + \\
\hline-1.17 & - & - & - & - & - & $\ldots$ & - & - & - & 0 & + & + \\
\hline-1.04 & - & - & - & - & - & $\ldots$ & - & - & 0 & 0 & + & + \\
\hline-0.92 & - & - & - & - & - & $\ldots$ & - & - & - & 0 & 0 & + \\
\hline-0.76 & - & - & - & - & - & $\ldots$ & - & - & - & 0 & + & + \\
\hline-0.62 & - & - & - & - & - & $\ldots$ & - & - & - & 0 & + & + \\
\hline-0.49 & - & - & - & - & - & $\ldots$ & - & - & - & 0 & + & + \\
\hline-0.35 & - & - & - & - & - & $\ldots$ & - & - & - & - & + & + \\
\hline-0.12 & - & - & - & - & - & $\ldots$ & - & - & - & - & + & + \\
\hline 0.07 & - & - & - & - & - & $\ldots$ & - & - & - & - & 0 & + \\
\hline 0.34 & - & - & - & - & - & $\ldots$ & - & - & - & - & 0 & + \\
\hline 0.68 & - & - & - & - & - & $\ldots$ & - & - & - & - & 0 & + \\
\hline 1.05 & - & - & - & - & - & $\ldots$ & - & - & - & - & - & + \\
\hline 5.92 & - & - & - & - & - & $\ldots$ & - & - & - & - & - & 0 \\
\hline
\end{tabular}

Notes: $\mathrm{Sx}=1, \mathrm{Sy}=1$

A negative sign indicates that the Domincan Republic's dominance surface is significantly above Colombia's, a positive sign indicates the opposite, and a zero indicates that the difference is not statistically significant. The ellipses indicate that all intervening signs are negative. 
Table 4: $t$-statistics for difference between household income with child allowances $v s$. with social security (Romania)

\begin{tabular}{|c|c|c|c|c|c|c|}
\hline \multicolumn{7}{|c|}{ Household income $\backslash$ Household size } \\
\hline & 6 or more & 5 or more & 4 or more & 3 or more & 2 or more & 1 or more \\
\hline 36,316 & -30.51 & -26.01 & -20.24 & -9.68 & 21.25 & 32.80 \\
\hline 46,630 & -36.27 & -30.34 & -24.34 & -11.96 & 20.14 & 31.48 \\
\hline 59,874 & -41.95 & -36.41 & -29.30 & -15.76 & 18.02 & 27.29 \\
\hline 76,880 & -47.80 & -41.96 & -34.84 & -20.38 & 13.75 & 19.26 \\
\hline 98,716 & -54.91 & -47.82 & -39.52 & -24.29 & 7.39 & 9.47 \\
\hline 126,750 & -57.50 & -50.75 & -42.30 & -27.13 & 0.45 & 1.75 \\
\hline 162,750 & -59.59 & -52.29 & -45.60 & -30.02 & -10.08 & -8.35 \\
\hline 208,980 & -47.90 & -45.00 & -42.05 & -29.21 & -15.98 & -13.77 \\
\hline 268,340 & -38.35 & -36.73 & -35.02 & -27.07 & -17.62 & -15.56 \\
\hline 344,550 & -27.02 & -25.99 & -25.41 & -19.47 & -13.52 & -11.95 \\
\hline 442,410 & -17.74 & -18.26 & -17.04 & -13.60 & -8.63 & -7.41 \\
\hline 568,070 & -18.13 & -11.28 & -10.25 & -7.50 & -4.46 & -3.76 \\
\hline 729,420 & -7.23 & -7.55 & -7.58 & -7.01 & -2.68 & -2.29 \\
\hline 936,590 & -4.30 & -3.70 & -3.26 & -1.81 & -0.25 & -0.23 \\
\hline $1,202,600$ & -10.34 & -5.66 & -3.48 & -1.65 & -0.07 & -0.06 \\
\hline $1,544,200$ & -7.86 & -3.89 & -2.17 & -1.23 & 0.37 & 0.33 \\
\hline
\end{tabular}

Notes: $\quad s=1$. Results are similar for $s=2$ and $s=3$.

A negative sign indicates that income with child allowances dominates 
Table 5: $t$-statistics for difference between per capita expenditures for literate and illiterate Peruvians, 1994 vs. 1985

$\begin{array}{rrr}\text { Household income } \backslash \text { Literacy } \\ & \text { Illiterate } & \text { Literate } \\ 403 & -1.95 & -3.21 \\ 518 & -4.93 & -5.76 \\ 665 & -7.69 & -8.35 \\ 854 & -14.93 & -15.33 \\ 1,097 & -22.37 & -24.37 \\ 1,408 & -28.97 & -31.28 \\ 1,808 & -35.47 & -38.95 \\ 2,322 & -41.48 & -46.19 \\ 2,981 & -46.16 & -51.91 \\ 3,828 & -48.38 & -53.91 \\ 4,915 & -49.63 & -55.40 \\ 6,311 & -46.49 & -51.90 \\ 8,103 & -40.41 & -45.30 \\ 10,405 & -35.02 & -39.00 \\ 13,360 & -26.61 & -29.54 \\ 17,154 & -21.45 & -23.74 \\ 22,026 & -16.02 & -17.51\end{array}$

Notes: $\quad \mathrm{s}=1$.

A negative sign indicates that household

expenditures in 1985 dominate

those in 1994, and vice-versa. 